Article

\title{
Optimal Multi-Step-Ahead Prediction of ARCH/GARCH Models and NoVaS Transformation
}

\author{
Jie Chen and Dimitris N. Politis * \\ Department of Mathematics, University of California, San Diego, CA 92093, USA \\ * Correspondence: dpolitis@ucsd.edu
}

Received: 31 October 2018; Accepted: 21 July 2019; Published: 8 August 2019

\begin{abstract}
This paper gives a computer-intensive approach to multi-step-ahead prediction of volatility in financial returns series under an ARCH/GARCH model and also under a model-free setting, namely employing the NoVaS transformation. Our model-based approach only assumes i.i.d innovations without requiring knowledge/assumption of the error distribution and is computationally straightforward. The model-free approach is formally quite similar, albeit a GARCH model is not assumed. We conducted a number of simulations to show that the proposed approach works well for both point prediction (under $L_{1}$ and / or $L_{2}$ measures) and prediction intervals that were constructed using bootstrapping. The performance of GARCH models and the model-free approach for multi-step ahead prediction was also compared under different data generating processes.
\end{abstract}

Keywords: bootstrap; $L_{1}$ and $L_{2}$ measures; $\operatorname{GARCH}(1,1)$; NoVaS transformation; multi-step prediction; Monte Carlo simulation

\section{Introduction}

Multi-step-ahead prediction in a time series amounts to predicting a sequence of future values using only the values observed over a finite time interval. Examples of time series for which multi-step-ahead prediction is useful include crop yields, stock prices, traffic volume, and electrical power consumption. In the paper at hand, we focus on multi-step-ahead prediction of squared financial returns, which is related to the so-called volatility, i.e., the conditional expectation of the squared returns. A typical approach to solve this problem, known as multi-stage or iterated prediction, is to construct a single model from the past observed time series data and then apply the model step by step to predict its future values. The iterated method uses the predicted value of the current time step to determine its value in the next time step. However, empirical evidence points to the fact that multi-stage prediction is susceptible to the error accumulation problem, i.e., errors committed in the past are propagated into future predictions.

The benchmark model for financial returns has been the GARCH(1,1); see Bollerslev et al. (1992) and the references therein. Notably, the work in Andersen et al. (2006), page 811, stated that, beyond the one-step-ahead case, there is no analytical form of the predictive density for multi-step-ahead predictions of volatility in GARCH models. However, the analytical form of the multi-step ahead predictive probability density function of a $\operatorname{GARCH}(1,1)$ process under Gaussian or Student $t$ innovations has been recently derived in the working paper of Abadir et al. (2018).

The work of Abadir et al. (2018) was an important breakthrough, but it hinged on knowing the error distribution that drove the $\mathrm{GARCH}(1,1)$ model. By contrast, the paper at hand proposes an alternative computer-intensive approach to multi-step-ahead prediction of the squared returns in $\mathrm{ARCH} / \mathrm{GARCH}$ and related models that does not require knowledge of the error distribution. Furthermore, an analogous methodology can be employed in a model-free setting using the normalizing and variance-stabilizing transformation (NoVaS) approach; for more details on NoVaS, 
see Politis $(2003,2007,2015)$. Notably, our method is not of the multi-stage/iterated type, and therefore eliminates the errors accumulation issue.

The remainder of the paper is organized as follows. Section 2 presents the proposed method for the optimal multi-step-ahead point predictions for ARCH/GARCH processes, as well as in a model-free setting using the NoVaS transformation. Section 3 addresses the associated methods for the construction of multi-step-ahead prediction intervals using bootstrapping. Section 4 illustrates the numerical performance of the proposed methods by means of simulated examples; some concluding remarks are provided in Section 5.

\section{Optimal Multi-Step-Ahead Point Prediction}

Consider data $X_{1}, \ldots, X_{n}$ from a zero mean and (strictly) stationary financial returns time series $\left\{X_{t}\right\}$. Our goal is to predict the future squared returns $X_{n+h}^{2}$ for any $h \geq 2$; the case $h=1$ was treated in Politis $(2007,2015)$.

Let $\mathcal{F}_{n}$ be a short-hand for the observed information set, i.e., $\mathcal{F}_{n}=\left\{X_{t}, 1 \leq t \leq n\right\}$. In the $L_{2}$ sense, the optimal predictor of $X_{n+h}^{2}$ based on $\mathcal{F}_{n}$ is the conditional mean and given by:

$$
\widehat{X_{n+h}^{2}}=E\left(X_{n+h}^{2} \mid \mathcal{F}_{n}\right) .
$$

Similarly, the optimal $L_{1}$ predictor is the conditional median:

$$
\widehat{X_{n+h}^{2}}=\operatorname{Median}\left(X_{n+h}^{2} \mid \mathcal{F}_{n}\right) \text {. }
$$

In the following parts of this section, we study the multi-step-ahead prediction in the nonlinear financial models ARCH/GARCH and in the NoVaS setting, which is an application of the model-free approach to financial returns data.

\section{1. $L_{2}$ Optimal Prediction for $A R C H(p)$ and $G A R C H(1,1)$ Models}

Suppose the data follow the $\mathrm{ARCH}(\mathrm{p})$ process of Engle (1982) defined by the recursion:

$$
X_{t}=\sigma_{t} \epsilon_{t}, \text { and } \sigma_{t}^{2}=\alpha+a_{1} X_{t-1}^{2}+\ldots+a_{p} X_{t-p}^{2}
$$

where $\alpha \geq 0, a_{j} \geq 0$ for all $j=1, \ldots, p$, and $\left\{\epsilon_{t}\right\} \sim$ i.i.d. $N(0,1)$.

First, consider the simplest case $h=2$. Based on ARCH(P) Model in (3), we can express $X_{n+1}$ and $X_{n+2}$ in the following way:

$$
\begin{gathered}
X_{n+1}=\epsilon_{n+1} \sqrt{\sigma_{n+1}^{2}} \text {, and } \sigma_{n+1}^{2}=\alpha+a_{1} X_{n}^{2}+\ldots+a_{p} X_{n-p+1}^{2}, \\
X_{n+2}=\epsilon_{n+2} \sqrt{\sigma_{n+2}^{2}} \text {, and } \sigma_{n+2}^{2}=\alpha+a_{1} X_{n+1}^{2}+\ldots+a_{p} X_{n-p+2}^{2} .
\end{gathered}
$$

Obviously, $X_{n+1}$ can be easily written as a function of the past observations $X_{n}, \ldots, X_{n+1-p}$ and the unknown future error $\epsilon_{n+1}$. Furthermore, we can also rewrite $X_{n+2}$ to be a function of $X_{n}, \ldots, X_{n+1-p}$ and the unknown future errors $\epsilon_{n+1}$ and $\epsilon_{n+2}$. The notations are as follows:

$$
\begin{aligned}
X_{n+1} & =\epsilon_{n+1} \sqrt{\alpha+a_{1} X_{n}+a_{2} X_{n-1}^{2}+\ldots+a_{p} X_{n-p+1}^{2}} \\
& =f_{1}\left(X_{n}, \ldots, X_{n-p+1} ; \epsilon_{n+1}\right)
\end{aligned}
$$


and:

$$
\begin{aligned}
X_{n+2} & =\epsilon_{n+2} \sqrt{\alpha+a_{1} X_{n+1}^{2}+a_{2} X_{n}^{2}+\ldots+a_{p} X_{n-p+2}^{2}} \\
& =\epsilon_{n+2} \sqrt{\alpha+a_{1} \epsilon_{n+1}^{2}\left(\alpha+a_{1} X_{n}+a_{2} X_{n-1}^{2}+\ldots+a_{p} X_{n-p+1}^{2}\right)+a_{2} X_{n}^{2}+\ldots+a_{p} X_{n-p+2}^{2}} \\
& =f_{2}\left(X_{n}, \ldots, X_{n-p+1} ; \epsilon_{n+1}, \epsilon_{n+2}\right) .
\end{aligned}
$$

Recursively, we can express $X_{n+h}$ for any $h \geq 1$ as a function of past observations $\left\{X_{1}, \ldots, X_{n}\right\}$ and the unknown future innovations $\left\{\epsilon_{n+1}, \ldots, \epsilon_{n+h}\right\}$ in the form:

$$
X_{n+h}=f_{h}\left(X_{1}, \ldots, X_{n} ; \epsilon_{n+1}, \ldots, \epsilon_{n+h}\right) .
$$

Since $\left\{X_{1}, \ldots, X_{n}\right\}$ are given and known, we can write (6) simply as:

$$
X_{n+h}=f_{h}\left(\epsilon_{n+1}, \ldots, \epsilon_{n+h}\right), \text { for any } h \geq 1
$$

The squared financial returns can be rewritten as $f_{h}^{2}$. Based on the assumption that $\epsilon_{t}$ is i.i.d $N(0,1)$, the conditional distribution function $F_{f_{h}^{2}}$ of the future squared returns $f_{h}^{2}(\cdot)$ can be derived. Hence, the optimal predictor (conditional median for $L_{1}$ or conditional mean for $L_{2}$ ) of $x_{n+h}^{2}$ is easy to calculate by $F_{f_{h}^{2}}$.

Take $h=1$ and $h=2$ as examples. By (4) and (5), the $L_{2}$ optimal predictors of $X_{n+1}^{2}$ and $X_{n+2}^{2}$ are:

$$
\begin{aligned}
\widehat{X_{n+1}^{2}} & =E\left\{\epsilon_{n+1}^{2}\left(\alpha+a_{1} X_{n}+a_{2} X_{n-1}^{2}+\ldots+a_{p} X_{n-p+1}^{2}\right) \mid \mathcal{F}_{n}\right\} \\
& =\alpha+a_{1} X_{n}^{2}+a_{2} X_{n-1}^{2}+\ldots+a_{p} X_{n-p+1}^{2}
\end{aligned}
$$

and:

$$
\begin{aligned}
\widehat{X_{n+2}^{2}} & =E\left[\epsilon_{n+2}^{2}\left(\alpha+a_{1} \sigma_{n+1}^{2} \epsilon_{n+1}^{2}+a_{2} X_{n}^{2}+\ldots+a_{p} X_{n-p+2}^{2}\right) \mid X_{1}, \ldots, X_{n}\right] \\
& =\alpha+a_{1} \sigma_{n+1}^{2}+a_{2} X_{n}^{2}+\ldots+a_{p} X_{n-p+2}^{2}
\end{aligned}
$$

since $E\left(\epsilon_{n+1}^{2} \mid \mathcal{F}_{n}\right)=1$ and $E\left(\epsilon_{n+2}^{2} \mid \mathcal{F}_{n}\right)=1$ by assumption. First, we can note that $\widehat{X_{n+1}^{2}}=f_{1}^{2}\left(\epsilon_{n+1}^{2}=1\right)$ and $\widehat{X_{n+2}^{2}}=f_{2}^{2}\left(\epsilon_{n+1}^{2}=1, \epsilon_{n+2}^{2}=1\right)$. Actually, we can easily verify that the $L_{2}$ optimal predictor for any $h \geq 1$ is given by:

$$
\widehat{X_{n+h}^{2}}=f_{h}^{2}\left(\epsilon_{n+1}^{2}=1, \ldots, \epsilon_{n+h}^{2}=1\right) .
$$

Note that because all $\epsilon_{t}$ 's are independent of each other, as well as of the past values of the $X$ series, the $h$-step-ahead predictor in (10) is equivalent to the method of multi-stage/iterated prediction that uses the predicted values of the current time step to determine its value in the next time step. However, for the $L_{1}$ case, because the median function is not a linear operator, this equivalence breaks down; see Section 2.3 in what follows.

Remark 1. As already mentioned, the benchmark for fitting financial returns is the $G A R C H(1,1)$. Nevertheless, a GARCH $(1,1)$ model is tantamount to an ARCH( $p)$ model with $p=\infty$ and an exponentially-decreasing coefficient; see, e.g., Francq and Zakoian (2011). Because of the exponentially decrease of the ARCH coefficients, it is customary to approximate the GARCH(1,1) models with an $A R C H(p)$ where $p$ is finite, albeit large. In this sense, all the above results apply verbatim to a $G A R C H(1,1)$ process as well. Of course, in fitting a $G A R C H(1,1)$ model, the GARCH equation is used to fit four parameters, which are then expanded to the $p$ coefficients of the approximating $A R C H(p)$ model. 


\section{2. $L_{2}$ Optimal Prediction for NoVaS}

Given a sequence of observations $\left\{X_{1}, \ldots, X_{n}\right\}$, we can fit the data by a special application of the model-free methodology, NoVaS, which was introduced by Politis $(2003,2007)$ for stationary data in prediction of squared financial returns. Let us continue considering a zero mean and (strictly) stationary financial return time series $\left\{X_{t}\right\}$. The NoVaS methodology is trying to map the dataset $X_{1}, \ldots, X_{n}$ to an i.i.d Gaussian dataset $\left\{W_{t}, t \leq n\right\}$.

The starting point is the ARCH model defined by:

$$
X_{t}=Z_{t} \sqrt{a+\sum_{i=1}^{p} a_{i} X_{t-i}^{2}}
$$

under which, the residual:

$$
\frac{X_{t}}{\sqrt{a+\sum_{i=1}^{p} a_{i} X_{t-i}^{2}}}
$$

is thought of as perfectly normalized and variance-stabilized, as it is assumed to be i.i.d.N $(0,1)$, which is actually not true here. This ratio can be interpreted as an attempt to "Studentize" the return $X_{t}$ by dividing with a time-localized measure of the standard deviation of $X_{t}$. However, there seems to be no reason to exclude the value of $X_{t}$ from an empirical, causal estimate of the standard deviation of $X_{t}$; recall that a causal estimate is one involving present and past data only, i.e., the data $\left\{X_{s}, s \leq t\right\}$.

Hence, the work in Politis (2003) defined a new "Studentized" quantity as follows:

$$
W_{t}:=\frac{X_{t}}{\sqrt{\alpha s_{t-1}^{2}+a_{0} X_{t}^{2}+\sum_{i=1}^{p} a_{i} X_{t-i}^{2}}} \text { for } t=p+1, p+2, \ldots, n \text {. }
$$

In the above, $s_{t-1}^{2}$ is an estimator of $\sigma_{X}^{2}=\operatorname{Var}\left(X_{1}\right)$ based on the data up to (but not including ${ }^{1}$ ) time $t$; under the zero mean assumption for $X_{1}$, the natural estimator is $s_{t-1}^{2}=(t-1)^{-1} \sum_{k=1}^{t-1} X_{k}^{2}$.

The definition in Equation (13) describes the proposed normalizing and variance-stabilizing transformation under which the data series $\left\{X_{t}\right\}$ is mapped to the new series $\left\{W_{t}\right\}$. The order $p(\geq 0)$ and the vector of nonnegative parameters $\left(\alpha, a_{0}, \ldots, a_{p}\right)$ are chosen by the practitioner with the twin goals of normalization and variance stabilization.

Furthermore, the NoVaS transformation Equation (13) can be re-arranged to yield:

$$
X_{t}=W_{t} \sqrt{\alpha s_{t-1}^{2}+a_{0} X_{t}^{2}+\sum_{i=1}^{p} a_{i} X_{t-i}^{2}} .
$$

Formally, the only real difference between the NoVaS of Equation (14) and the ARCH of Equation (11) is the presence of the term $X_{t}^{2}$ paired with the coefficient $a_{0}$. Replacing the term $a$ in Equation (11) by the term $\alpha s_{t-1}^{2}$ in Equation (14) is only natural since the former has, by necessity, units of variance; in other words, the term $a$ in Equation (11) is not scale invariant, whereas the term $\alpha$ in Equation (14) is.

Given the assumed structure of the return series, the target of variance stabilization, which amounts to constructing a local estimator of scale for Studentization purposes, requires:

$$
\alpha \geq 0, \quad a_{i} \geq 0 \text { for all } i \geq 0, \text { and } \alpha+\sum_{i=0}^{p} a_{i}=1
$$

1 The reason for not including time $t$ in the variance estimator is for purposes of notational clarity, as well as the easy identifiability of the effect of the coefficient $a_{0}$ associated with $X_{t}^{2}$ in the denominator of Equation (13). 
Equation (15) has the interesting implication that the $\left\{W_{t}\right\}$ series can be assumed to have a (unconditional) variance that is (approximately) unity. Nevertheless, note that $p$ and $\alpha, a_{0}, \ldots, a_{p}$ must be carefully chosen to achieve a degree of conditional homoscedasticity as well; to do this, one must necessarily take $p$ small enough, as well as $\alpha$ small enough or even equal to zero, so that a local (as opposed to global) estimator of scale is obtained. The work in Politis (2003) provided two structures for the $a_{i}$ coefficients satisfying Equation (15). One is to let $\alpha=0$ and $a_{i}=1 /(p+1)$ for all $0 \leq i \leq p$; this specification is called the simple NoVaS transformation and involves only one parameter, namely the order $p$, to be chosen by the practitioner. The other one is given by the exponential (decay) NoVaS, where $\alpha=0$ and $a_{i}=c^{\prime} e^{-c i}$ for all $0 \leq i \leq p$. The exponential scheme involves choosing two parameters: $p$ and $c>0$, since $c^{\prime}$ is determined by Equation (15). For more details of how to select the optimal parameters here, see Politis (2015).

The above Equation (14) can be used for one-step-ahead prediction in an analogous way to the $\mathrm{ARCH} / \mathrm{GARCH}$ models already discussed. In fact, Equation (14) is formally analogous to an $\mathrm{ARCH}(p)$ model with i.i.d. errors given by $\epsilon_{t}=\frac{W_{t}}{\sqrt{1-a_{0} W_{t}^{2}}}$. Hence, the construction of the previous subsection can be repeated to write $X_{n+h}$ as some function of $\left\{X_{1}, \ldots, X_{n}\right\}$ and $\left\{W_{t}, t=1, \ldots, h\right\}$ for any $h \geq 1$, i.e.,

$$
X_{n+h}=f_{h}\left(X_{1}, \ldots, X_{n} ; W_{n+1}, \ldots, W_{n+h}\right) .
$$

Since the data $\left\{X_{1}, \ldots, X_{n}\right\}$ are given, we can simplify (16) as:

$$
X_{n+h}=f_{h}\left(W_{n+1}, \ldots, W_{n+h}\right) .
$$

In the $L_{2}$ sense, the optimal predictor of $X_{n+h}^{2}$ based on $\mathcal{F}_{n}$ is given by:

$$
\left.\widehat{X_{n+h}^{2}}=E\left(X_{n+h}^{2} \mid \mathcal{F}_{n}\right)=E\left\{f_{h}^{2}\left(W_{n+1}, \ldots, W_{n+h}\right) \mid \mathcal{F}_{n}\right)\right\}
$$

Since the $W_{t}$ are i.i.d, we can get analogous results with those concerning the ARCH/GARCH models, i.e.,

$$
\left.\widehat{X_{n+h}^{2}}=f_{h}^{2}\left(W_{n+1}^{2}=1, \ldots, W_{n+h}^{2}=1\right)\right)
$$

Therefore, for any $h \geq 1$, we can use similar ideas to that in the ARCH/GARCH cases to conduct multi-step-ahead prediction in NoVaS by approximating the conditional mean or median from their conditional distribution functions.

\section{3. $L_{1}$ Optimal Prediction and Generalizations}

We can generalize the above prediction method to an interesting class of prediction functions $g(\cdot)$, namely the power family where $g(x)=x^{k}$ for some fixed $k$ and the power-absolute value family where $g(x)=|x|^{k}$. So far, we have worked on the prediction of $X_{n+h^{\prime}}^{2}$ that is $g(x)=x^{2}$. More generally, we can derive the best $L_{2}$ or $L_{1}$ predictor of $g\left(X_{n+h}\right)$ given $\mathcal{F}_{n}$.

Regarding $L_{1}$ optimal prediction, it was already mentioned that it is not equivalent to the multi-stage/iterated approach. For the $h=2$ case, we can easily get an analytic formula of the conditional distribution of $g\left(X_{n+h}\right)$ assuming an $\mathrm{ARCH}(p)$ model with normal errors. An approximate analytic form of the conditional distribution function of $X_{n+2}^{2}$ is given as follows:

$$
F_{f_{2}^{2}}\left(x \mid\left\{X_{n}, \ldots, X_{1}\right\}\right)=\frac{\gamma\left(\frac{1}{2}, \frac{x}{2 A}\right)}{B \sqrt{\pi}}, \quad x>0
$$

where:

$$
\begin{gathered}
A=\alpha+a_{2} X_{n}^{2}+a_{3} X_{n-1}^{2}+\ldots+a_{p} X_{n-p+2}^{2}, \\
B=a_{1} \sigma_{n+1}^{2}=a_{1}\left(\alpha+a_{1} X_{n}^{2}+\ldots+a_{p} X_{n-p+1}^{2}\right),
\end{gathered}
$$




$$
\gamma(s, x)=\int_{0}^{x} t^{s-1} \mathrm{e}^{-t} \mathrm{~d} t, \quad s>0 .
$$

Solving $F_{f_{h}^{2}}\left(x \mid\left\{X_{n}, \ldots, X_{1}\right\}\right)=\frac{1}{2}$, we obtain $\widehat{X_{n+2}^{2}} \approx \frac{\pi}{8} A B^{2}+\pi^{4} B^{4}$ for the $L_{1}$ optimal predictor.

The general case $h \geq 1$ has been recently worked out by Abadir et al. (2018). However, the analytical method crucially depends on the assumption for the error distribution. A more robust way to approximate the conditional distribution of $g\left(X_{n+h}\right)$ can be derived using bootstrapping ${ }^{2}$.

Before going into bootstrapping, note that a simple Monte Carlo simulation can re-produce the analytical calculations in a straightforward manner. For example, if the ARCH/GARCH errors are assumed independent with a particular distribution function $F_{\epsilon}$, we can easily generate pseudo-replicates of $X_{n+h}$ by using Equation (7), and simulating many sets of $\left\{\epsilon_{n+1}, \ldots, \epsilon_{n+h}\right\}$ where each $\epsilon_{t}$ is drawn i.i.d from $F_{\epsilon}$.

If $F_{\epsilon}$ has a known structural form with some unknown parameters, e.g., Student- $t$ with unknown degrees of freedom, one can use a data-based estimate of the unknown parameter in order to estimate $F_{\epsilon}$ and then proceed with the simulation; this is then equivalent to a parametric bootstrap procedure. In the more realistic case where $F_{\epsilon}$ is treated as unknown, it can be estimated by the empirical distribution of the ARCH/GARCH residuals and then used in the simulation; this is equivalent to the standard (nonparametric) bootstrap. Because of the formal analogy of NoVaS to ARCH models, a similar bootstrap method works for NoVaS as well; this is the so-called model-free bootstrap. Detailed Algorithms 1-5 are given in the following two subsections.

\subsection{Bootstrap Algorithms for ARCH/GARCH Point Prediction}

Assume an ARCH/GARCH model with all parameters known and errors $\left\{\epsilon_{t}\right\} \sim$ i.i.d. with known distribution $F_{\epsilon}$. Under the independence of $\left\{\epsilon_{t}\right\}$ for all $t \geq 1$, we can generate many $\epsilon_{n+1}^{*}, \ldots, \epsilon_{n+h}^{*} \sim$ i.i.d. from $F_{\epsilon}$ by Monte Carlo simulation and compute many pseudo-values of the quantity of interest $g\left(X_{n+h}^{*}\right)$.

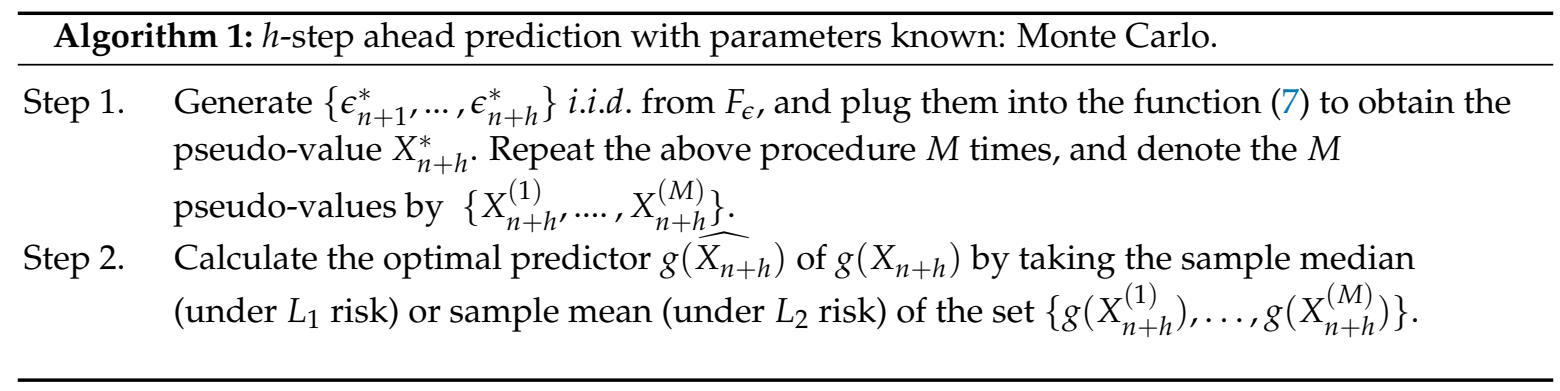

If the parameters and $F_{\epsilon}$ in the ARCH model are unknown, estimates must be used, in which case the Monte Carlo simulation becomes bootstrapping. Let $\hat{F}_{\epsilon}$ denote the estimator of $F_{\epsilon}$. The two cases, parametric and nonparametric bootstrap, depend on whether the parametric form of $F_{\epsilon}$ is known or not. In the former case, $\hat{F}_{\epsilon}$ uses the parametric form of $F_{\epsilon}$ with parameters estimated and plugged in. In the latter, $\hat{F}_{\epsilon}$ is typically taken to be the empirical distribution of the $\mathrm{ARCH}$ residuals normalized to unit variance.

2 The bootstrap validity is not shown in this paper, because it is beyond the scope of the paper. 


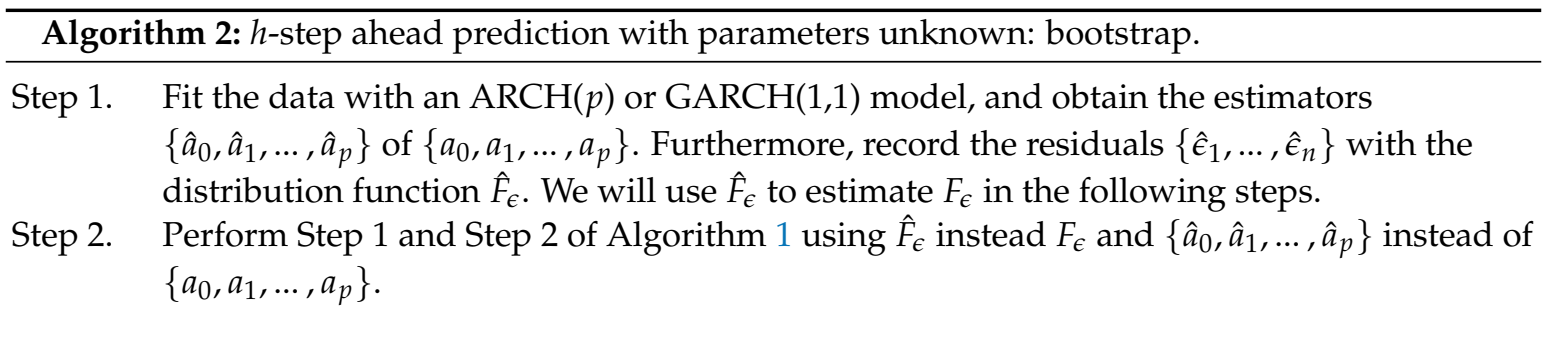

Remark 2. The work in Bose and Mukherjee (2009) proposed a weighted linear estimator (WLE) to estimate the ARCH parameters. This method does not involve nonlinear optimization and gives a closed-form expression, so it is computationally easier to obtain the estimator compared to maximum likelihood. In our numerical work, we used the WLE to obtain the estimators $\left\{\hat{a}_{0}, \hat{a}_{1}, \ldots, \hat{a}_{p}\right\}$ of $\left\{a_{0}, a_{1}, \ldots, a_{p}\right\}$ in Algorithm 2.

\subsection{Bootstrap Algorithms for NoVaS-Based Point Prediction}

We now go back to the model-free setting of Section 2.2. In order to estimate the conditional mean or conditional median in the NoVaS setting, we should first use one of the NoVaS methods (simple vs. exponential, generalized or not, etc.) to obtain the coefficients $\alpha, a_{0}, a_{1}, \ldots, a_{p}$. Based on the independence of the $W_{t}$, we can use Monte Carlo and/or bootstrap to generate different $W_{n+k}^{*}$ for $k=1, \ldots, h$, and consequently approximate the distribution of $f_{h}\left(W_{n+1}, \ldots, W_{n+h}\right)$. Denote by $\hat{F}_{W}$ the empirical distribution of the transformed data $W_{p+1}, \ldots, W_{n}$. Similar to Algorithm 2, we can use either a (truncated $)^{3}$ standard normal distribution or $\hat{F}_{W}$ to generate the pseudo-values $W_{n+k}^{*}$.

Algorithm 3: $h$-step ahead prediction for NoVaS: bootstrap.

Step 1. Use one of the NoVaS methods (simple vs. exponential, generalized or not, etc.) to obtain the transformed data $\left\{W_{t}\right.$ for $\left.t=p+1, \ldots, n\right\}$ and the coefficients $\alpha, p$, and $a_{0}, a_{1}, \ldots, a_{p}$.

Step 2. Compute the analytic form of Equation (16), i.e., express $X_{n+h}$ as a function of $\left\{X_{1}, \ldots, X_{n}\right\}$ and $\left\{W_{n+1}, \ldots, W_{n+h}\right\}$ using the values $\left\{a_{0}, a_{1}, \ldots, a_{p}\right\}$ obtained in Step 1.

Step 3. Generate $\left\{W_{n+1}^{*}, \ldots, W_{n+h}^{*}\right\}$ as i.i.d. either from a (truncated) standard normal distribution or from $\hat{F}_{W}$, and plug them into the function (16) to obtain the pseudo-value $X_{n+h}^{*}$. Repeat the above procedure $M$ times and denote the $M$ pseudo-values by $\left\{X_{n+h^{\prime}}^{(1)} \ldots, X_{n+h}^{(M)}\right\}$.

Step 4. Calculate the optimal predictor $g\left(\widehat{X_{n+h}}\right)$ of $g\left(X_{n+h}\right)$ by taking the sample median (under $L_{1}$ risk) or sample mean (under $L_{2}$ risk) of the set $\left\{g\left(X_{n+h}^{(1)}\right), \ldots, g\left(X_{n+h}^{(M)}\right)\right\}$.

\section{Optimal Multi-Step-Ahead Prediction Intervals}

Going beyond point prediction, it may be desirable to construct prediction intervals for $g\left(X_{n+h}\right)$ with a target coverage level $(1-\beta) 100 \%$. One-step ahead prediction intervals have been discussed in detail in Pan and Politis (2016); see also Chapter 10 of Politis (2015). In this section, we will propose a construction of multi-step ahead prediction intervals in the given setting of financial returns data $\left\{X_{1}, \ldots, X_{n}\right\}$.

3 From (13), it follows that:

$$
\frac{1}{W_{t}^{2}}=\frac{\alpha s_{t-1}^{2}+a_{0} X_{t}^{2}+\sum_{i=1}^{p} a_{i} X_{t-i}^{2}}{X_{t}^{2}} \geq a_{0}
$$

since all the parameters are nonnegative; thus, $\left|W_{t}\right| \leq 1 / \sqrt{a_{0}}$, i.e., the range of the $W_{t}$ is finite. Typically, the $\left\{W_{t}\right\}$ variables have a large enough range such that the boundedness is not seen as spoiling the normality from a practical perspective, but in any theoretical works and/or simulations, it is necessary to use the standard normal distribution truncated to the range $\pm 1 / \sqrt{a_{0}}$. 
As explained in Politis (2015), the bootstrap is a sine qua non for the construction of prediction intervals as it allows us to incorporate the variability of estimated quantities in our estimate of the conditional distribution of $g\left(X_{n+h}\right)$ given $\left\{X_{1}, \ldots, X_{n}\right\}$. The variability of estimated quantities is not so important in point prediction when only the center of the conditional distribution is of interest. However, it is crucial in order to obtain an estimate of the conditional distribution that is not too narrow, yielding prediction intervals with accurate coverage.

In what follows, we give the algorithms for bootstrap prediction intervals for $g\left(X_{n+h}\right)$ in the two settings: model-based for ARCH/GARCH models and model-free based on NoVaS. The algorithms follow the "forward bootstrap" paradigm introduced in Pan and Politis (2016).

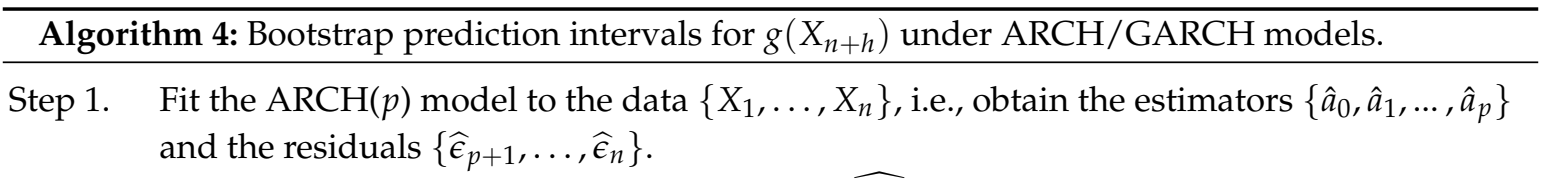

Step 2. Use Algorithm 1 or Algorithm 2 to compute $g\left(\widehat{X_{n+h}}\right)$, the point predictor of $g\left(X_{n+h}\right)$ of choice.

Step 3. (a) Re-sample (with replacement) the residuals $\left\{\widehat{\epsilon}_{p+1}, \ldots, \widehat{\epsilon}_{n}\right\}$ to create the pseudo-errors $\epsilon_{p+1}^{*}, \cdots, \epsilon_{n}^{*}$ and $\epsilon_{n+1}^{*}, \cdots, \epsilon_{n+h}^{*}$.

(b) Let $\left(X_{1}^{*}, \ldots, X_{p}^{*}\right)^{\prime}=\left(X_{1+I}, \cdots, X_{p+I}\right)^{\prime}$ where $I$ is generated as a discrete random variable uniform on the values $0,1, \ldots, n-p$. Now, use the fitted ARCH model of Step 1 to generate bootstrap pseudo-data $X_{t}^{*}$ for $t=p+1, \ldots, n$ in a recursive manner.

(c) Based on the bootstrap data $X_{1}^{*}, \ldots, X_{n}^{*}$, re-estimate the parameters obtaining $\left\{\hat{a}_{0}^{*}, \hat{a}_{1}^{*}, \ldots, \hat{a}_{p}^{*}\right\}$.

(d) Re-define the last $p$ values of the bootstrap data to match the original, i.e., re-define $X_{t}^{*}=X_{t}$ for $t=n-p+1, \ldots, n$; this is the "forward bootstrap" 4 construction.

(e) Use the fitted ARCH model of Step 1, the bootstrap data $X_{1}^{*}, \ldots, X_{n}^{*}$, and the pseudo-errors $\epsilon_{n+1}^{*}, \cdots, \epsilon_{n+h}^{*}$ to generate recursively the future bootstrap data $X_{t}^{*}$ for $t=n+1, \ldots, n+h$.

(f). Based on the bootstrap data $X_{n-p+1}^{*}, \ldots, X_{n}^{*}$ and the re-estimated parameters $\left\{\hat{a}_{0}^{*}, \hat{a}_{1}^{*}, \ldots, \hat{a}_{p}^{*}\right\}$, use Algorithm 1 or 2 (according to which one was used in Step 2 to calculate the bootstrap predictor denoted by $g\left(\widehat{X_{n+h}^{*}}\right)$.

(g) Calculate the bootstrap $\operatorname{root}^{5}: g\left(X_{n+h}^{*}\right)-g\left(\widehat{X_{n+h}^{*}}\right)$.

Step 4. Repeat Step 3 above $B$ times; the $B$ bootstrap root replicates are collected in an empirical distribution whose $\alpha$-quantile is denoted $q(\alpha)$. The $(1-\beta) 100 \%$ equal-tailed prediction interval for $g\left(X_{n+h}\right)$ is then given by $\left[g\left(\widehat{X_{n+h}}\right)+q(\beta / 2), g\left(\widehat{X_{n+h}}\right)+q(1-\beta / 2)\right]$.

4 Most of the bootstrap methods will not have their last $p$ values $X_{t}^{*}$ where $t=n, \ldots, n-p+1$, exactly equal to the original values as needed for the prediction process. Herein lies the problem, since the behavior of the predictors for future values needs to be captured conditionally on the original values. In this forward bootstrap step, we redefine the last $p$ values of $X_{t}^{*}$ and make them equal to the original ones; see Pan and Politis (2014).

5 Since we do not have much information of the distribution of $g\left(X_{n+h}\right)-g\left(\widehat{X_{n+h}}\right)$, we could use the distribution of $g\left(X_{n+h}^{*}\right)-g\left(\widehat{X_{n+h}^{*}}\right)$ to approximate it. Therefore, we can employ the quantile of the approximated distribution to calculate the prediction intervals in the following steps. See more theoretical inference in Politis (2015). 


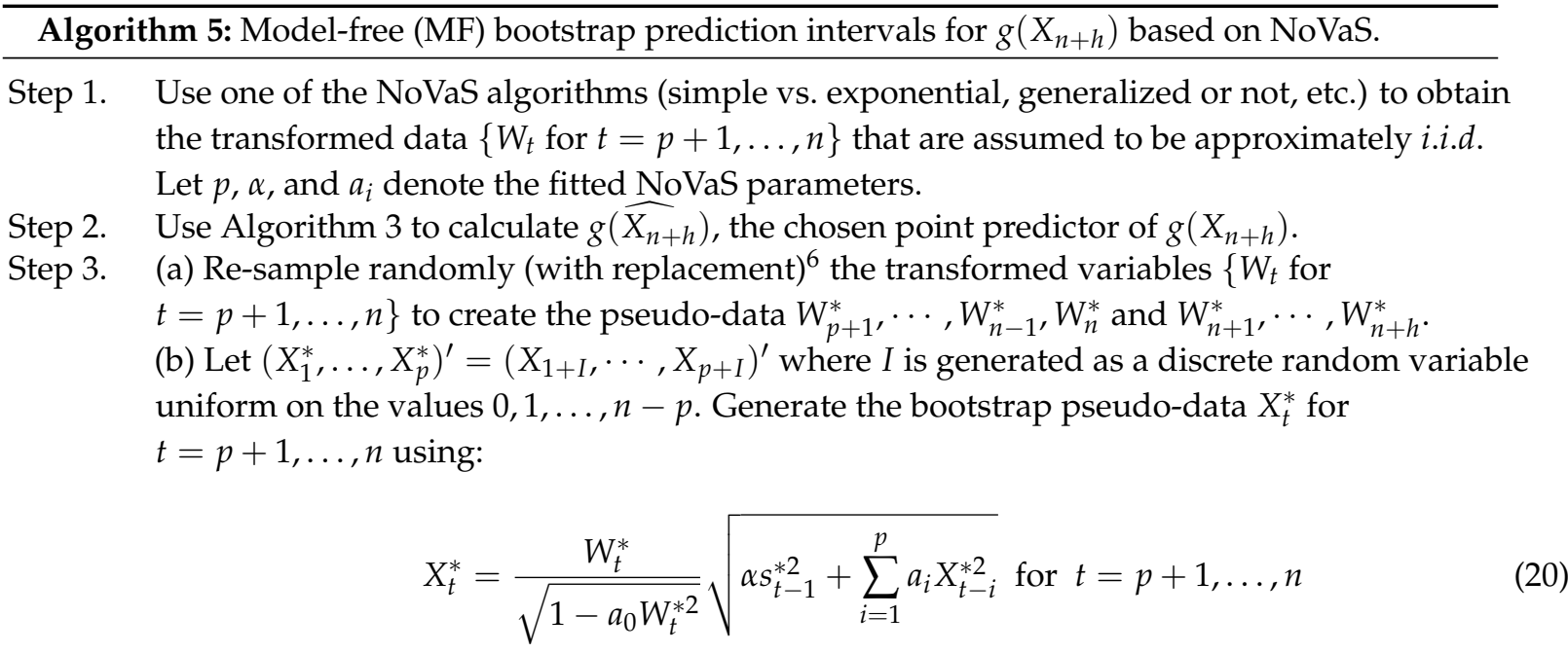

where $s_{t-1}^{* 2}=(t-1)^{-1} \sum_{k=1}^{t-1} X_{k}^{* 2}$.

(c) Based on the bootstrap data $X_{1}^{*}, \ldots, X_{n}^{*}$, re-estimate the NoVaS parameters, obtaining $p^{*}, \alpha^{*}$, and $a_{i}^{*}$; for simplicity, we can keep the same value for $p$, i.e., let $p^{*}$ equal $p$.

(d) Re-define the last $p$ values of the bootstrap data to match the original, i.e., re-define $X_{t}^{*}=X_{t}$ for $t=n-p+1, \ldots, n$; this is the "forward bootstrap" construction.

(e) Calculate the bootstrap future value $X_{n+h}^{*}$ by iteration as:

$$
X_{n+1}^{*}=\frac{W_{n+1}^{*}}{\sqrt{1-a_{0} W_{n+1}^{* 2}}} \sqrt{\alpha s_{n}^{2}+\sum_{i=1}^{p} a_{i} X_{n-i+1}^{2}}
$$

where $s_{n}^{2}=n^{-1} \sum_{i=1}^{n} X_{i}^{2}$. If $h<p$, for $j=2, \ldots, h$ :

$$
X_{n+j}^{*}=\frac{W_{n+j}^{*}}{\sqrt{1-a_{0} W_{n+j}^{* 2}}} \sqrt{\alpha s_{n+1-j}^{2}+\sum_{k=1}^{j-1} a_{k} X_{n-k+j}^{* 2}+\sum_{i=j}^{p} a_{i} X_{n-i+j}^{2}}
$$

If $h \geq p$, for $j=2, \ldots, h$ :

$$
X_{n+j}^{*}=\frac{W_{n+j}^{*}}{\sqrt{1-a_{0} W_{n+j}^{* 2}}} \sqrt{\alpha s_{n+1-j}^{2}+\sum_{i=1}^{p} a_{i} X_{n-i+j}^{* 2}}
$$

where $s_{n+1-j}^{2}=(n+j-1)^{-1}\left(\sum_{i=1}^{n} X_{i}^{2}+\sum_{k=1}^{j-1} X_{n+k}^{* 2}\right)$.

(f) Based on the bootstrap data $X_{n-p+1}^{*}, \ldots, X_{n}^{*}$ and the parameters $p^{*}, \alpha^{*}, a_{0}^{*}, a_{1}^{*}, \ldots, a_{p}^{*}$, use Algorithm 3 to calculate the bootstrap predictor $g\left(\widehat{X_{n+h}^{*}}\right)$.

(g) Calculate the bootstrap root: $g\left(X_{n+h}^{*}\right)-g\left(\widehat{X_{n+h}^{*}}\right)$.

Step 4. Repeat Step 3 above $B$ times; the $B$ bootstrap root replicates are collected in an empirical distribution whose $\alpha$-quantile is denoted $q(\alpha)$. The $(1-\beta) 100 \%$ equal-tailed prediction interval for $g\left(X_{n+h}\right)$ is given by $\left[g\left(\widehat{X_{n+h}}\right)+q(\beta / 2), g\left(\widehat{X_{n+h}}\right)+q(1-\beta / 2)\right]$.

6 It is also possible to use other bootstrap methods, for example wild bootstrap or block bootstrap, to create pseudo-data. It depends on the information you have for $W_{t}$. 


\section{Simulations and Finite Sample Performance}

In this section, we conduct simulations to examine the finite sample performance of our algorithms.

\subsection{Settings}

In the simulation, 200 datasets $X_{n}=\left(X_{1}, \ldots, X_{n}\right)^{\prime}$, each of size $n=100$, are generated separately by the following seven different $\mathrm{GARCH}(1,1)$ models.

Model 1. Standard GARCH with Gaussian errors and finite fourth moment: $X_{t}=\sigma_{t} \epsilon_{t}, \sigma_{t}^{2}=0.00001+0.73 \sigma_{t-1}^{2}+0.10 X_{t-1}^{2},\left\{\epsilon_{t}\right\} \sim$ i.i.d. $N(0,1)$.

Model 2. Standard GARCH with Gaussian errors and infinite fourth moment: $X_{t}=\sigma_{t} \epsilon_{t}, \sigma_{t}^{2}=0.00001+0.8895 \sigma_{t-1}^{2}+0.10 X_{t-1}^{2},\left\{\epsilon_{t}\right\} \sim$ i.i.d. $N(0,1)$.

Model 3. Standard GARCH with Student- $t$ errors: $X_{t}=\sigma_{t} \epsilon_{t}, \sigma_{t}^{2}=0.00001+0.73 \sigma_{t-1}^{2}+0.10 X_{t-1}^{2},\left\{\epsilon_{t}\right\} \sim$ i.i.d. $t$ distributed with five degrees of freedom. Model 4. GARCH with time-varying parameters (TV-GARCH):

The value of $\beta$ decreases as a linear function of $t$, starting at $\beta_{1}=0.10$ for $t=1$, and ending at $\beta=0.05$ for $t=n$. At the same time, the value of $\alpha$ increases as a linear function of $t$, starting at $\alpha=0.73$ for $t=1$, and ending at $\alpha=0.93$ for $t=n . \omega=0.00001$ and $\left\{\epsilon_{t}\right\} \sim$ i.i.d. $N(0,1)$.

Model 5. Two-state Markov Switching GARCH(1,1) (MS-GARCH):

$$
X_{t}=\sigma_{t} \epsilon_{t}, \sigma_{t}^{2}=\sum_{s=1}^{2} 1\left\{P\left(S_{t}=s\right)\right\}\left[\omega_{s}+\alpha_{t} \sigma_{t-1}^{2}+\beta_{s} X_{t-1}^{2}\right]
$$

In the first regime, we set $\alpha_{1}=0.9, \beta_{1}=0.07, \omega_{1}=2.4 e-5$. In the second regime, we set $\alpha_{2}=0.7$, $\beta_{2}=0.22, \omega_{2}=1.2 e-4$. The transition probabilities for the first regime are $p_{11}=0.9$ and $p_{12}=0.1$, while for the second regime, we use $p_{21}=0.3$ and $p_{22}=0.7 .\left\{\epsilon_{t}\right\} \sim$ i.i.d. $N(0,1)$.

Model 6. Smooth transition GARCH (ST-GARCH):

$$
X_{t}=[a-b(t / T)] \sigma_{t} \epsilon_{t}, \sigma_{t}^{2}=\omega+\alpha \sigma_{t-1}^{2}+\beta X_{t-1}^{2}
$$

where $\left\{\epsilon_{t}\right\} \sim$ i.i.d. $N(0,1) . \omega=1.2 e-5, \alpha=0.9, \beta=0.07, a=\alpha+\beta=0.97$, and $b=\beta / \alpha \approx 0.078$.

Model 7. Stochastic volatility model (SV-GARCH):

$$
\begin{gathered}
X_{t} \mid h_{t} \sim N\left(0, \exp \left(h_{t}\right)\right), \\
h_{t} \mid h_{t-1} \sim N\left(\mu+\phi\left(h_{t-1}-\mu\right), \eta^{2}\right), h_{0} \sim N\left(\mu, \eta^{2} /\left(1-\phi^{2}\right)\right),
\end{gathered}
$$

where $\mu=-10, \phi=0.95, \eta=0.2$.

We performed up to five-step-ahead point predictions and interval predictions for each dataset. We used $M=5000$ simulations to compute the point predictions. For the bootstrap prediction intervals, we used $B=300$ replications; we would have liked to use a higher number of bootstrap replications, but it was practically infeasible having 200 simulated datasets in each of which the point predictors were computed in a computer-intensive way. However, a practitioner having a single dataset at hand could (and should) use a higher $B$, e.g., $B=1000$ or more.

Five models or transformations were used to fit the data in both point predictions and interval predictions as follows: fitting a GARCH $(1,1)$ model, simple-NoVaS, exponential NoVaS (Exp-NoVaS), generalized simple NoVaS (GS-NoVaS), and generalized exponential NoVaS (GE-NoVaS).

In point predictions, the mean absolute deviations (MAD) and mean squared errors (MSE) for five-step-ahead point predictions in both the $L_{1}$ and $L_{2}$ sense (the absolute value or the square of the prediction error at the updated time point averaged over the 200 replications) were recorded. Furthermore, the bootstrap prediction interval $\left(L_{i}, U_{i}\right)$ with a nominal coverage $95 \%$ was constructed for the future values $X_{n+h}$ with $h=1, \ldots, 5$. 
The corresponding empirical average coverage level (CVR) and the average length (LEN) of the constructed intervals and the standard error (St.err) associated with each length of the constructed intervals are calculated as:

$$
\begin{gathered}
C V R=\frac{1}{N} \sum_{i=1}^{N} \mathbf{1}_{\left[L_{i}, U_{i}\right]} X_{(n+h, i)} \\
L E N=\frac{1}{N} \sum_{i=1}^{N} L E N_{i} \text { and St.err }=\sqrt{\frac{1}{N} \sum_{i=1}^{N}\left(L E N_{i}-L E N\right)^{2}}
\end{gathered}
$$

where $L E N_{i}=U_{i}-L_{i}$.

\subsection{Results and Discussions}

The simulation results for point predictions are shown in Tables 1-28. The following conclusions can be obtained from the results:

- When comparing the MADs between the $L_{1}$ and $L_{2}$ predictions by fitting the same models, we can find that the MADs of $L_{1}$ predictions were always smaller than those of $L_{2}$ predictions. Furthermore, we can find that the MSEs of $L_{1}$ predictions were always bigger than those of $L_{2}$ predictions, when comparing the MSEs between the $L_{1}$ and $L_{2}$ predictions with the same model settings. This was expected since in the $L_{1}$ sense, we tried to minimize the mean absolute deviations, while for $L_{2}$, the loss function to be minimized was the mean squared error.

- Furthermore, for each model's fitting results, there were no obvious error accumulation problems in the multi-step-ahead prediction for both $L_{1}$ and $L_{2}$ measures.

- NoVaS methods consistently performed better than $\operatorname{GARCH}(1,1)$ for all data generating processes. When the prediction step $h$ was higher, the difference of their respective performances became smaller.

Table 1. MADs of $L_{1}$ predictions for data generated from $\operatorname{GARCH}(1,1)$ with $\omega=0.00001, \alpha=$ $0.8895, \theta=0.10$, and $\left\{\epsilon_{t}\right\} \sim$ i.i.d. $N(0,1)$. Exp, exponential; GS, generalized simple; GE, generalized exponential.

\begin{tabular}{cccccc}
\hline Prediction Step & $\mathbf{1}$ & $\mathbf{2}$ & $\mathbf{3}$ & $\mathbf{4}$ & $\mathbf{5}$ \\
\hline Fitting a GARCH & $8.23 \times 10^{-5}$ & $7.35 \times 10^{-5}$ & $6.93 \times 10^{-5}$ & $8.86 \times 10^{-5}$ & $1.49 \times 10^{-4}$ \\
Simple-NoVaS & $6.99 \times 10^{-5}$ & $7.88 \times 10^{-5}$ & $8.29 \times 10^{-5}$ & $1.06 \times 10^{-4}$ & $1.63 \times 10^{-4}$ \\
Exp-NoVaS & $7.21 \times 10^{-5}$ & $8.28 \times 10^{-5}$ & $8.72 \times 10^{-5}$ & $1.14 \times 10^{-4}$ & $1.70 \times 10^{-4}$ \\
GS-NoVaS & $6.30 \times 10^{-5}$ & $7.31 \times 10^{-5}$ & $8.07 \times 10^{-5}$ & $8.71 \times 10^{-5}$ & $9.94 \times 10^{-5}$ \\
GE-NoVaS & $7.02 \times 10^{-5}$ & $8.44 \times 10^{-5}$ & $8.76 \times 10^{-5}$ & $1.16 \times 10^{-4}$ & $1.71 \times 10^{-4}$ \\
\hline
\end{tabular}

Table 2. MADs of $L_{2}$ predictions for data generated from $\operatorname{GARCH}(1,1)$ with $\omega=0.00001, \alpha=$ $0.8895, \theta=0.10$, and $\left\{\epsilon_{t}\right\} \sim$ i.i.d. $N(0,1)$.

\begin{tabular}{cccccc}
\hline Prediction Step & $\mathbf{1}$ & $\mathbf{2}$ & $\mathbf{3}$ & $\mathbf{4}$ & $\mathbf{5}$ \\
\hline Fitting a GARCH & $1.48 \times 10^{-4}$ & $2.07 \times 10^{-4}$ & $2.67 \times 10^{-4}$ & $3.20 \times 10^{-4}$ & $4.60 \times 10^{-4}$ \\
Simple-NoVaS & $5.22 \times 10^{-5}$ & $6.44 \times 10^{-5}$ & $6.61 \times 10^{-5}$ & $8.82 \times 10^{-5}$ & $1.49 \times 10^{-4}$ \\
Exp-NoVaS & $5.13 \times 10^{-5}$ & $6.41 \times 10^{-5}$ & $6.58 \times 10^{-5}$ & $8.84 \times 10^{-5}$ & $1.50 \times 10^{-4}$ \\
GS-NoVaS & $4.71 \times 10^{-5}$ & $5.97 \times 10^{-5}$ & $6.06 \times 10^{-5}$ & $8.48 \times 10^{-5}$ & $1.46 \times 10^{-4}$ \\
GE-NoVaS & $4.84 \times 10^{-5}$ & $6.26 \times 10^{-5}$ & $6.38 \times 10^{-5}$ & $8.73 \times 10^{-5}$ & $1.48 \times 10^{-4}$ \\
\hline
\end{tabular}


Table 3. MSEs of $L_{1}$ predictions for data generated from GARCH(1,1) with $\omega=0.00001, \alpha=0.8895, \theta=$ 0.10 , and $\left\{\epsilon_{t}\right\} \sim$ i.i.d. $N(0,1)$.

\begin{tabular}{cccccc}
\hline Prediction Step & $\mathbf{1}$ & $\mathbf{2}$ & $\mathbf{3}$ & $\mathbf{4}$ & $\mathbf{5}$ \\
\hline Fitting a GARCH & $2.48 \times 10^{-8}$ & $4.42 \times 10^{-8}$ & $4.36 \times 10^{-8}$ & $1.94 \times 10^{-7}$ & $1.12 \times 10^{-6}$ \\
Simple-NoVaS & $1.23 \times 10^{-8}$ & $4.24 \times 10^{-8}$ & $4.17 \times 10^{-8}$ & $1.94 \times 10^{-7}$ & $1.12 \times 10^{-6}$ \\
Exp-NoVaS & $1.18 \times 10^{-8}$ & $4.21 \times 10^{-8}$ & $4.14 \times 10^{-8}$ & $1.94 \times 10^{-7}$ & $1.12 \times 10^{-6}$ \\
GS-NoVaS & $1.08 \times 10^{-8}$ & $4.09 \times 10^{-8}$ & $3.94 \times 10^{-8}$ & $1.92 \times 10^{-7}$ & $1.13 \times 10^{-6}$ \\
GE-NoVaS & $1.10 \times 10^{-8}$ & $4.14 \times 10^{-8}$ & $4.06 \times 10^{-8}$ & $1.92 \times 10^{-7}$ & $1.12 \times 10^{-6}$ \\
\hline
\end{tabular}

Table 4. MSEs of $L_{2}$ predictions for data generated from $\operatorname{GARCH}(1,1)$ with $\omega=0.00001, \alpha=0.8895, \theta=$ 0.10 , and $\left\{\epsilon_{t}\right\} \sim$ i.i.d. $N(0,1)$.

\begin{tabular}{cccccc}
\hline Prediction Step & $\mathbf{1}$ & $\mathbf{2}$ & $\mathbf{3}$ & $\mathbf{4}$ & $\mathbf{5}$ \\
\hline Fitting a GARCH & $1.03 \times 10^{-7}$ & $2.11 \times 10^{-7}$ & $3.92 \times 10^{-7}$ & $6.26 \times 10^{-7}$ & $1.86 \times 10^{-6}$ \\
Simple-NoVaS & $1.36 \times 10^{-8}$ & $4.15 \times 10^{-8}$ & $4.10 \times 10^{-8}$ & $1.93 \times 10^{-7}$ & $1.11 \times 10^{-6}$ \\
Exp-NoVaS & $1.31 \times 10^{-8}$ & $4.13 \times 10^{-8}$ & $4.07 \times 10^{-8}$ & $1.93 \times 10^{-7}$ & $1.12 \times 10^{-6}$ \\
GS-NoVaS & $1.03 \times 10^{-8}$ & $3.88 \times 10^{-8}$ & $3.73 \times 10^{-8}$ & $1.91 \times 10^{-7}$ & $1.13 \times 10^{-6}$ \\
GE-NoVaS & $1.13 \times 10^{-8}$ & $3.98 \times 10^{-8}$ & $3.93 \times 10^{-8}$ & $1.88 \times 10^{-7}$ & $1.11 \times 10^{-6}$ \\
\hline
\end{tabular}

Table 5. MADs of $L_{1}$ predictions for data generated from $\operatorname{GARCH}(1,1)$ with $\omega=0.00001, \alpha=0.73, \theta=$ 0.10 , and $\left\{\epsilon_{t}\right\} \sim$ i.i.d. $N(0,1)$.

\begin{tabular}{cccccc}
\hline Prediction Step & $\mathbf{1}$ & $\mathbf{2}$ & $\mathbf{3}$ & $\mathbf{4}$ & $\mathbf{5}$ \\
\hline Fitting a GARCH & $4.84 \times 10^{-5}$ & $4.69 \times 10^{-5}$ & $5.18 \times 10^{-5}$ & $5.45 \times 10^{-5}$ & $5.99 \times 10^{-5}$ \\
Simple-NoVaS & $4.94 \times 10^{-5}$ & $4.73 \times 10^{-5}$ & $5.26 \times 10^{-5}$ & $5.59 \times 10^{-5}$ & $6.00 \times 10^{-5}$ \\
Exp-NoVaS & $4.87 \times 10^{-5}$ & $4.69 \times 10^{-5}$ & $5.24 \times 10^{-5}$ & $5.55 \times 10^{-5}$ & $6.00 \times 10^{-5}$ \\
GS-NoVaS & $4.83 \times 10^{-5}$ & $4.67 \times 10^{-5}$ & $5.17 \times 10^{-5}$ & $5.44 \times 10^{-5}$ & $5.94 \times 10^{-5}$ \\
GE-NoVaS & $4.84 \times 10^{-5}$ & $4.69 \times 10^{-5}$ & $5.25 \times 10^{-5}$ & $5.43 \times 10^{-5}$ & $6.00 \times 10^{-5}$ \\
\hline
\end{tabular}

Table 6. MADs of $L_{2}$ predictions for data generated from $\operatorname{GARCH}(1,1)$ with $\omega=0.00001, \alpha=0.73, \theta=$ 0.10 , and $\left\{\epsilon_{t}\right\} \sim$ i.i.d. $N(0,1)$.

\begin{tabular}{cccccc}
\hline Prediction Step & $\mathbf{1}$ & $\mathbf{2}$ & $\mathbf{3}$ & $\mathbf{4}$ & $\mathbf{5}$ \\
\hline Fitting a GARCH & $5.68 \times 10^{-5}$ & $5.45 \times 10^{-5}$ & $5.54 \times 10^{-5}$ & $6.25 \times 10^{-5}$ & $0.26 \times 10^{-5}$ \\
Simple-NoVaS & $6.20 \times 10^{-5}$ & $6.05 \times 10^{-5}$ & $6.06 \times 10^{-5}$ & $6.96 \times 10^{-5}$ & $6.67 \times 10^{-5}$ \\
Exp-NoVaS & $6.20 \times 10^{-5}$ & $6.09 \times 10^{-5}$ & $6.15 \times 10^{-5}$ & $7.05 \times 10^{-5}$ & $6.87 \times 10^{-5}$ \\
GS-NoVaS & $5.98 \times 10^{-5}$ & $5.81 \times 10^{-5}$ & $5.84 \times 10^{-5}$ & $6.55 \times 10^{-5}$ & $6.37 \times 10^{-5}$ \\
GE-NoVaS & $5.50 \times 10^{-5}$ & $5.30 \times 10^{-5}$ & $5.75 \times 10^{-5}$ & $6.22 \times 10^{-5}$ & $6.23 \times 10^{-5}$ \\
\hline
\end{tabular}

Table 7. MSEs of $L_{1}$ predictions for data generated from $\operatorname{GARCH}(1,1)$ with $\omega=0.00001, \alpha=0.73, \theta=$ 0.10 , and $\left\{\epsilon_{t}\right\} \sim$ i.i.d. $N(0,1)$.

\begin{tabular}{cccccc}
\hline Prediction step & 1 & 2 & 3 & 4 & 5 \\
\hline Fitting a GARCH & $7.94 \times 10^{-9}$ & $8.48 \times 10^{-9}$ & $7.80 \times 10^{-9}$ & $8.67 \times 10^{-9}$ & $1.03 \times 10^{-8}$ \\
Simple-NoVaS & $8.00 \times 10^{-9}$ & $8.35 \times 10^{-9}$ & $7.85 \times 10^{-9}$ & $8.88 \times 10^{-9}$ & $1.03 \times 10^{-8}$ \\
Exp-NoVaS & $7.95 \times 10^{-9}$ & $8.33 \times 10^{-9}$ & $7.83 \times 10^{-9}$ & $8.84 \times 10^{-9}$ & $1.03 \times 10^{-8}$ \\
GS-NoVaS & $7.78 \times 10^{-9}$ & $8.35 \times 10^{-9}$ & $7.63 \times 10^{-9}$ & $8.59 \times 10^{-9}$ & $1.02 \times 10^{-8}$ \\
GE-NoVaS & $8.02 \times 10^{-9}$ & $8.64 \times 10^{-9}$ & $7.99 \times 10^{-9}$ & $8.92 \times 10^{-9}$ & $1.02 \times 10^{-8}$ \\
\hline
\end{tabular}


Table 8. MSEs of $L_{2}$ predictions for data generated from $\operatorname{GARCH}(1,1)$ with $\omega=0.00001, \alpha=0.73, \theta=$ 0.10 , and $\left\{\epsilon_{t}\right\} \sim$ i.i.d. $N(0,1)$.

\begin{tabular}{cccccc}
\hline Prediction Step & $\mathbf{1}$ & $\mathbf{2}$ & $\mathbf{3}$ & $\mathbf{4}$ & $\mathbf{5}$ \\
\hline Fitting a GARCH & $7.28 \times 10^{-9}$ & $7.47 \times 10^{-9}$ & $6.50 \times 10^{-9}$ & $7.64 \times 10^{-9}$ & $8.65 \times 10^{-9}$ \\
Simple-NoVaS & $7.79 \times 10^{-9}$ & $7.50 \times 10^{-9}$ & $6.84 \times 10^{-9}$ & $8.67 \times 10^{-9}$ & $8.85 \times 10^{-9}$ \\
Exp-NoVaS & $7.48 \times 10^{-9}$ & $7.34 \times 10^{-9}$ & $6.64 \times 10^{-9}$ & $8.39 \times 10^{-9}$ & $8.70 \times 10^{-9}$ \\
GS-NoVaS & $7.12 \times 10^{-9}$ & $7.40 \times 10^{-9}$ & $6.41 \times 10^{-9}$ & $7.90 \times 10^{-9}$ & $8.43 \times 10^{-9}$ \\
GE-NoVaS & $6.99 \times 10^{-9}$ & $7.44 \times 10^{-9}$ & $6.43 \times 10^{-9}$ & $7.68 \times 10^{-9}$ & $8.53 \times 10^{-9}$ \\
\hline
\end{tabular}

Table 9. MADs of $L_{1}$ predictions for data generated from $\operatorname{GARCH}(1,1)$ with $\omega=0.00001, \alpha=0.73, \theta=$ 0.10 , and $\left\{\epsilon_{t}\right\} \sim$ i.i.d. $t$ distributed with degrees of freedom of 5.

\begin{tabular}{cccccc}
\hline Prediction Step & $\mathbf{1}$ & $\mathbf{2}$ & $\mathbf{3}$ & $\mathbf{4}$ & $\mathbf{5}$ \\
\hline Fitting a GARCH & $1.46 \times 10^{-4}$ & $1.26 \times 10^{-4}$ & $1.29 \times 10^{-4}$ & $1.84 \times 10^{-4}$ & $1.77 \times 10^{-4}$ \\
Simple-NoVaS & $1.44 \times 10^{-4}$ & $1.24 \times 10^{-4}$ & $1.27 \times 10^{-4}$ & $1.82 \times 10^{-4}$ & $1.76 \times 10^{-4}$ \\
Exp-NoVaS & $1.43 \times 10^{-4}$ & $1.22 \times 10^{-4}$ & $1.26 \times 10^{-4}$ & $1.80 \times 10^{-4}$ & $1.75 \times 10^{-4}$ \\
GS-NoVaS & $1.43 \times 10^{-4}$ & $1.23 \times 10^{-4}$ & $1.27 \times 10^{-4}$ & $1.80 \times 10^{-4}$ & $1.76 \times 10^{-4}$ \\
GE-NoVaS & $1.43 \times 10^{-4}$ & $1.23 \times 10^{-4}$ & $1.29 \times 10^{-4}$ & $1.81 \times 10^{-4}$ & $1.77 \times 10^{-4}$ \\
\hline
\end{tabular}

Table 10. MADs of $L_{2}$ predictions for data generated from $\operatorname{GARCH}(1,1)$ with $\omega=0.00001, \alpha=0.73, \theta=$ 0.10 , and $\left\{\epsilon_{t}\right\} \sim$ i.i.d. $t$ distributed with degrees of freedom of 5 .

\begin{tabular}{cccccc}
\hline Prediction Step & $\mathbf{1}$ & $\mathbf{2}$ & $\mathbf{3}$ & $\mathbf{4}$ & $\mathbf{5}$ \\
\hline Fitting a GARCH & $1.72 \times 10^{-4}$ & $1.53 \times 10^{-4}$ & $1.63 \times 10^{-4}$ & $2.17 \times 10^{-4}$ & $2.16 \times 10^{-4}$ \\
Simple-NoVaS & $1.58 \times 10^{-4}$ & $1.35 \times 10^{-4}$ & $1.44 \times 10^{-4}$ & $1.97 \times 10^{-4}$ & $1.92 \times 10^{-4}$ \\
Exp-NoVaS & $1.57 \times 10^{-4}$ & $1.35 \times 10^{-4}$ & $1.46 \times 10^{-4}$ & $1.98 \times 10^{-4}$ & $1.93 \times 10^{-4}$ \\
GS-NoVaS & $1.56 \times 10^{-4}$ & $1.35 \times 10^{-4}$ & $1.45 \times 10^{-4}$ & $1.99 \times 10^{-4}$ & $1.92 \times 10^{-4}$ \\
GE-NoVaS & $1.59 \times 10^{-4}$ & $1.34 \times 10^{-4}$ & $1.48 \times 10^{-4}$ & $2.01 \times 10^{-4}$ & $1.94 \times 10^{-4}$ \\
\hline
\end{tabular}

Table 11. MSEs of $L_{1}$ predictions for data generated from GARCH(1,1) with $\omega=0.00001, \alpha=0.73, \theta=$ 0.10 , and $\left\{\epsilon_{t}\right\} \sim$ i.i.d. $t$ distributed with degrees of freedom of 5.

\begin{tabular}{cccccc}
\hline Prediction Step & $\mathbf{1}$ & $\mathbf{2}$ & $\mathbf{3}$ & $\mathbf{4}$ & $\mathbf{5}$ \\
\hline Fitting a GARCH & $1.06 \times 10^{-7}$ & $7.11 \times 10^{-8}$ & $8.97 \times 10^{-8}$ & $2.95 \times 10^{-7}$ & $3.81 \times 10^{-7}$ \\
Simple-NoVaS & $1.03 \times 10^{-7}$ & $6.86 \times 10^{-8}$ & $9.00 \times 10^{-8}$ & $2.93 \times 10^{-7}$ & $3.83 \times 10^{-7}$ \\
Exp-NoVaS & $1.03 \times 10^{-7}$ & $6.83 \times 10^{-8}$ & $9.00 \times 10^{-8}$ & $2.93 \times 10^{-7}$ & $3.82 \times 10^{-7}$ \\
GS-NoVaS & $1.03 \times 10^{-7}$ & $6.90 \times 10^{-8}$ & $9.05 \times 10^{-8}$ & $2.94 \times 10^{-7}$ & $3.84 \times 10^{-7}$ \\
GE-NoVaS & $1.05 \times 10^{-7}$ & $6.88 \times 10^{-8}$ & $9.23 \times 10^{-8}$ & $2.96 \times 10^{-7}$ & $3.85 \times 10^{-7}$ \\
\hline
\end{tabular}

Table 12. MSEs of $L_{2}$ predictions for data generated from $\operatorname{GARCH}(1,1)$ with $\omega=0.00001, \alpha=0.73, \theta=$ 0.10 , and $\left\{\epsilon_{t}\right\} \sim$ i.i.d. $t$ distributed with degrees of freedom of 5 .

\begin{tabular}{cccccc}
\hline Prediction Step & $\mathbf{1}$ & $\mathbf{2}$ & $\mathbf{3}$ & $\mathbf{4}$ & $\mathbf{5}$ \\
\hline Fitting a GARCH & $1.05 \times 10^{-7}$ & $6.94 \times 10^{-8}$ & $8.91 \times 10^{-8}$ & $2.84 \times 10^{-7}$ & $3.79 \times 10^{-7}$ \\
Simple-NoVaS & $9.24 \times 10^{-8}$ & $6.03 \times 10^{-8}$ & $8.32 \times 10^{-8}$ & $2.77 \times 10^{-7}$ & $3.66 \times 10^{-7}$ \\
Exp-NoVaS & $9.16 \times 10^{-8}$ & $5.96 \times 10^{-8}$ & $8.28 \times 10^{-8}$ & $2.76 \times 10^{-7}$ & $3.64 \times 10^{-7}$ \\
GS-NoVaS & $9.17 \times 10^{-8}$ & $6.07 \times 10^{-8}$ & $8.35 \times 10^{-8}$ & $2.77 \times 10^{-7}$ & $3.67 \times 10^{-7}$ \\
GE-NoVaS & $9.48 \times 10^{-8}$ & $6.12 \times 10^{-8}$ & $8.53 \times 10^{-8}$ & $2.80 \times 10^{-7}$ & $3.67 \times 10^{-7}$ \\
\hline
\end{tabular}


Table 13. MADs of $L_{1}$ predictions for data generated from $\operatorname{GARCH}(1,1)$ with slowing-varying parameters (TV-GARCH).

\begin{tabular}{cccccc}
\hline Prediction Step & $\mathbf{1}$ & $\mathbf{2}$ & $\mathbf{3}$ & $\mathbf{4}$ & $\mathbf{5}$ \\
\hline Fitting a GARCH & $1.94 \times 10^{-4}$ & $2.17 \times 10^{-4}$ & $2.01 \times 10^{-4}$ & $1.76 \times 10^{-4}$ & $2.09 \times 10^{-4}$ \\
Simple-NoVaS & $1.91 \times 10^{-4}$ & $2.12 \times 10^{-4}$ & $2.03 \times 10^{-4}$ & $1.72 \times 10^{-4}$ & $2.07 \times 10^{-4}$ \\
Exp-NoVaS & $1.91 \times 10^{-4}$ & $2.12 \times 10^{-4}$ & $2.02 \times 10^{-4}$ & $1.72 \times 10^{-4}$ & $2.06 \times 10^{-4}$ \\
GS-NoVaS & $1.91 \times 10^{-4}$ & $2.13 \times 10^{-4}$ & $2.02 \times 10^{-4}$ & $1.73 \times 10^{-4}$ & $2.06 \times 10^{-4}$ \\
GE-NoVaS & $1.97 \times 10^{-4}$ & $2.17 \times 10^{-4}$ & $2.02 \times 10^{-4}$ & $1.79 \times 10^{-4}$ & $2.13 \times 10^{-4}$ \\
\hline
\end{tabular}

Table 14. MADs of $L_{2}$ predictions for data generated from $\operatorname{GARCH}(1,1)$ with slowing-varying parameters (TV-GARCH).

\begin{tabular}{cccccc}
\hline Prediction Step & $\mathbf{1}$ & $\mathbf{2}$ & $\mathbf{3}$ & $\mathbf{4}$ & $\mathbf{5}$ \\
\hline Fitting a GARCH & $1.90 \times 10^{-4}$ & $2.13 \times 10^{-4}$ & $2.03 \times 10^{-4}$ & $1.73 \times 10^{-4}$ & $2.02 \times 10^{-4}$ \\
Simple-NoVaS & $1.94 \times 10^{-4}$ & $2.11 \times 10^{-4}$ & $2.07 \times 10^{-4}$ & $1.74 \times 10^{-4}$ & $2.10 \times 10^{-4}$ \\
Exp-NoVaS & $1.94 \times 10^{-4}$ & $2.11 \times 10^{-4}$ & $2.05 \times 10^{-4}$ & $1.74 \times 10^{-4}$ & $2.09 \times 10^{-4}$ \\
GS-NoVaS & $1.91 \times 10^{-4}$ & $2.10 \times 10^{-4}$ & $1.99 \times 10^{-4}$ & $1.71 \times 10^{-4}$ & $2.03 \times 10^{-4}$ \\
GE-NoVaS & $1.89 \times 10^{-4}$ & $2.09 \times 10^{-4}$ & $1.99 \times 10^{-4}$ & $1.72 \times 10^{-4}$ & $2.07 \times 10^{-4}$ \\
\hline
\end{tabular}

Table 15. MSEs of $L_{1}$ predictions for data generated from $\operatorname{GARCH}(1,1)$ with slowing-varying parameters (TV-GARCH).

\begin{tabular}{cccccc}
\hline Prediction Step & $\mathbf{1}$ & $\mathbf{2}$ & $\mathbf{3}$ & $\mathbf{4}$ & $\mathbf{5}$ \\
\hline Fitting a GARCH & $1.21 \times 10^{-7}$ & $1.70 \times 10^{-7}$ & $1.28 \times 10^{-7}$ & $1.06 \times 10^{-7}$ & $1.58 \times 10^{-7}$ \\
Simple-NoVaS & $1.15 \times 10^{-7}$ & $1.61 \times 10^{-7}$ & $1.23 \times 10^{-7}$ & $9.86 \times 10^{-8}$ & $1.53 \times 10^{-7}$ \\
Exp-NoVaS & $1.16 \times 10^{-7}$ & $1.61 \times 10^{-7}$ & $1.23 \times 10^{-7}$ & $9.95 \times 10^{-8}$ & $1.52 \times 10^{-7}$ \\
GS-NoVaS & $1.16 \times 10^{-7}$ & $1.62 \times 10^{-7}$ & $1.24 \times 10^{-7}$ & $9.99 \times 10^{-8}$ & $1.53 \times 10^{-7}$ \\
GE-NoVaS & $1.25 \times 10^{-7}$ & $1.70 \times 10^{-7}$ & $1.31 \times 10^{-7}$ & $1.08 \times 10^{-7}$ & $1.61 \times 10^{-7}$ \\
\hline
\end{tabular}

Table 16. MSEs of $L_{2}$ predictions for data generated from $\operatorname{GARCH}(1,1)$ with slowing-varying parameters (TV-GARCH).

\begin{tabular}{cccccc}
\hline Prediction Step & $\mathbf{1}$ & $\mathbf{2}$ & $\mathbf{3}$ & $\mathbf{4}$ & $\mathbf{5}$ \\
\hline Fitting a GARCH & $1.07 \times 10^{-7}$ & $1.55 \times 10^{-7}$ & $1.12 \times 10^{-7}$ & $9.44 \times 10^{-8}$ & $1.41 \times 10^{-7}$ \\
Simple-NoVaS & $9.60 \times 10^{-8}$ & $1.41 \times 10^{-7}$ & $1.06 \times 10^{-7}$ & $8.36 \times 10^{-8}$ & $1.32 \times 10^{-7}$ \\
Exp-NoVaS & $9.60 \times 10^{-8}$ & $1.40 \times 10^{-7}$ & $1.05 \times 10^{-7}$ & $8.39 \times 10^{-8}$ & $1.30 \times 10^{-7}$ \\
GS-NoVaS & $9.70 \times 10^{-8}$ & $1.41 \times 10^{-7}$ & $1.07 \times 10^{-7}$ & $8.51 \times 10^{-8}$ & $1.32 \times 10^{-7}$ \\
GE-NoVaS & $1.04 \times 10^{-7}$ & $1.47 \times 10^{-7}$ & $1.12 \times 10^{-7}$ & $9.02 \times 10^{-8}$ & $1.38 \times 10^{-7}$ \\
\hline
\end{tabular}

Table 17. MADs of $L_{1}$ predictions for data generated from two-state Markov switching GARCH(1,1) (MS-GARCH).

\begin{tabular}{cccccc}
\hline Prediction Step & $\mathbf{1}$ & $\mathbf{2}$ & $\mathbf{3}$ & $\mathbf{4}$ & $\mathbf{5}$ \\
\hline Fitting a GARCH & $6.76 \times 10^{-4}$ & $7.67 \times 10^{-4}$ & $8.44 \times 10^{-4}$ & $7.80 \times 10^{-4}$ & $7.14 \times 10^{-4}$ \\
Simple-NoVaS & $7.00 \times 10^{-4}$ & $7.74 \times 10^{-4}$ & $8.85 \times 10^{-4}$ & $7.90 \times 10^{-4}$ & $7.26 \times 10^{-4}$ \\
Exp-NoVaS & $7.02 \times 10^{-4}$ & $7.75 \times 10^{-4}$ & $8.87 \times 10^{-4}$ & $7.92 \times 10^{-4}$ & $7.26 \times 10^{-4}$ \\
GS-NoVaS & $6.97 \times 10^{-4}$ & $7.70 \times 10^{-4}$ & $8.80 \times 10^{-4}$ & $7.91 \times 10^{-4}$ & $7.23 \times 10^{-4}$ \\
GE-NoVaS & $7.06 \times 10^{-4}$ & $7.75 \times 10^{-4}$ & $8.85 \times 10^{-4}$ & $7.98 \times 10^{-4}$ & $7.27 \times 10^{-4}$ \\
\hline
\end{tabular}


Table 18. MADs of $L_{2}$ predictions for data generated from two-state Markov switching GARCH $(1,1)$ (MS-GARCH).

\begin{tabular}{cccccc}
\hline Prediction Step & $\mathbf{1}$ & $\mathbf{2}$ & $\mathbf{3}$ & $\mathbf{4}$ & $\mathbf{5}$ \\
\hline Fitting a GARCH & $7.80 \times 10^{-4}$ & $9.01 \times 10^{-4}$ & $9.21 \times 10^{-4}$ & $9.17 \times 10^{-4}$ & $8.80 \times 10^{-4}$ \\
Simple-NoVaS & $6.77 \times 10^{-4}$ & $7.50 \times 10^{-4}$ & $8.59 \times 10^{-4}$ & $7.73 \times 10^{-4}$ & $7.10 \times 10^{-4}$ \\
Exp-NoVaS & $6.78 \times 10^{-4}$ & $7.50 \times 10^{-4}$ & $8.61 \times 10^{-4}$ & $7.75 \times 10^{-4}$ & $7.09 \times 10^{-4}$ \\
GS-NoVaS & $6.76 \times 10^{-4}$ & $7.50 \times 10^{-4}$ & $8.59 \times 10^{-4}$ & $7.76 \times 10^{-4}$ & $7.08 \times 10^{-4}$ \\
GE-NoVaS & $6.81 \times 10^{-4}$ & $7.50 \times 10^{-4}$ & $8.62 \times 10^{-4}$ & $7.79 \times 10^{-4}$ & $7.09 \times 10^{-4}$ \\
\hline
\end{tabular}

Table 19. MSEs of $L_{1}$ predictions for data generated from two-state Markov switching GARCH(1,1) (MS-GARCH).

\begin{tabular}{cccccc}
\hline Prediction Step & $\mathbf{1}$ & $\mathbf{2}$ & $\mathbf{3}$ & $\mathbf{4}$ & $\mathbf{5}$ \\
\hline Fitting a GARCH & $1.27 \times 10^{-6}$ & $2.30 \times 10^{-6}$ & $2.35 \times 10^{-6}$ & $2.78 \times 10^{-6}$ & $1.49 \times 10^{-6}$ \\
Simple-NoVaS & $1.45 \times 10^{-6}$ & $2.46 \times 10^{-6}$ & $2.73 \times 10^{-6}$ & $3.09 \times 10^{-6}$ & $1.76 \times 10^{-6}$ \\
Exp-NoVaS & $1.45 \times 10^{-6}$ & $2.46 \times 10^{-6}$ & $2.73 \times 10^{-6}$ & $3.10 \times 10^{-6}$ & $1.76 \times 10^{-6}$ \\
GS-NoVaS & $1.43 \times 10^{-6}$ & $2.45 \times 10^{-6}$ & $2.71 \times 10^{-6}$ & $3.10 \times 10^{-6}$ & $1.74 \times 10^{-6}$ \\
GE-NoVaS & $1.46 \times 10^{-6}$ & $2.47 \times 10^{-6}$ & $2.74 \times 10^{-6}$ & $3.12 \times 10^{-6}$ & $1.76 \times 10^{-6}$ \\
\hline
\end{tabular}

Table 20. MSEs of $L_{2}$ predictions for data generated from two-state Markov switching GARCH $(1,1)$ (MS-GARCH).

\begin{tabular}{cccccc}
\hline Prediction Step & $\mathbf{1}$ & $\mathbf{2}$ & $\mathbf{3}$ & $\mathbf{4}$ & $\mathbf{5}$ \\
\hline Fitting a GARCH & $1.29 \times 10^{-6}$ & $2.28 \times 10^{-6}$ & $2.05 \times 10^{-6}$ & $2.55 \times 10^{-6}$ & $1.51 \times 10^{-6}$ \\
Simple-NoVaS & $1.34 \times 10^{-6}$ & $2.33 \times 10^{-6}$ & $2.58 \times 10^{-6}$ & $2.97 \times 10^{-6}$ & $1.64 \times 10^{-6}$ \\
Exp-NoVaS & $1.34 \times 10^{-6}$ & $2.33 \times 10^{-6}$ & $2.59 \times 10^{-6}$ & $2.98 \times 10^{-6}$ & $1.64 \times 10^{-6}$ \\
GS-NoVaS & $1.33 \times 10^{-6}$ & $2.32 \times 10^{-6}$ & $2.57 \times 10^{-6}$ & $2.97 \times 10^{-6}$ & $1.62 \times 10^{-6}$ \\
GE-NoVaS & $1.35 \times 10^{-6}$ & $2.33 \times 10^{-6}$ & $2.59 \times 10^{-6}$ & $2.99 \times 10^{-6}$ & $1.64 \times 10^{-6}$ \\
\hline
\end{tabular}

Table 21. MADs of $L_{1}$ predictions for data generated from smooth transition GARCH(1,1) (ST-GARCH).

\begin{tabular}{cccccc}
\hline Prediction Step & $\mathbf{1}$ & $\mathbf{2}$ & $\mathbf{3}$ & $\mathbf{4}$ & $\mathbf{5}$ \\
\hline Fitting a GARCH & $1.83 \times 10^{-4}$ & $1.78 \times 10^{-4}$ & $2.01 \times 10^{-4}$ & $2.02 \times 10^{-4}$ & $2.22 \times 10^{-4}$ \\
Simple-NoVaS & $1.83 \times 10^{-4}$ & $1.79 \times 10^{-4}$ & $2.03 \times 10^{-4}$ & $2.02 \times 10^{-4}$ & $2.24 \times 10^{-4}$ \\
Exp-NoVaS & $1.82 \times 10^{-4}$ & $1.78 \times 10^{-4}$ & $2.03 \times 10^{-4}$ & $2.02 \times 10^{-4}$ & $2.24 \times 10^{-4}$ \\
GS-NoVaS & $1.81 \times 10^{-4}$ & $1.78 \times 10^{-4}$ & $2.02 \times 10^{-4}$ & $1.98 \times 10^{-4}$ & $2.21 \times 10^{-4}$ \\
GE-NoVaS & $1.82 \times 10^{-4}$ & $1.79 \times 10^{-4}$ & $2.05 \times 10^{-4}$ & $1.99 \times 10^{-4}$ & $2.24 \times 10^{-4}$ \\
\hline
\end{tabular}

Table 22. MADs of $L_{2}$ predictions for data generated from smooth transition GARCH(1,1) (ST-GARCH).

\begin{tabular}{cccccc}
\hline Prediction Step & $\mathbf{1}$ & $\mathbf{2}$ & $\mathbf{3}$ & $\mathbf{4}$ & $\mathbf{5}$ \\
\hline Fitting a GARCH & $2.23 \times 10^{-4}$ & $2.14 \times 10^{-4}$ & $2.18 \times 10^{-4}$ & $2.39 \times 10^{-4}$ & $2.42 \times 10^{-4}$ \\
Simple-NoVaS & $1.91 \times 10^{-4}$ & $1.88 \times 10^{-4}$ & $2.02 \times 10^{-4}$ & $2.09 \times 10^{-4}$ & $2.19 \times 10^{-4}$ \\
Exp-NoVaS & $1.89 \times 10^{-4}$ & $1.86 \times 10^{-4}$ & $2.03 \times 10^{-4}$ & $2.09 \times 10^{-4}$ & $2.19 \times 10^{-4}$ \\
GS-NoVaS & $1.90 \times 10^{-4}$ & $1.89 \times 10^{-4}$ & $2.03 \times 10^{-4}$ & $2.09 \times 10^{-4}$ & $2.17 \times 10^{-4}$ \\
GE-NoVaS & $1.86 \times 10^{-4}$ & $1.83 \times 10^{-4}$ & $2.06 \times 10^{-4}$ & $2.03 \times 10^{-4}$ & $2.20 \times 10^{-4}$ \\
\hline
\end{tabular}

Table 23. MSEs of $L_{1}$ predictions for data generated from smooth transition GARCH(1,1) (ST-GARCH)

\begin{tabular}{cccccc}
\hline Prediction Step & $\mathbf{1}$ & $\mathbf{2}$ & $\mathbf{3}$ & $\mathbf{4}$ & $\mathbf{5}$ \\
\hline Fitting a GARCH & $1.12 \times 10^{-7}$ & $1.23 \times 10^{-7}$ & $1.14 \times 10^{-7}$ & $1.13 \times 10^{-7}$ & $1.43 \times 10^{-7}$ \\
Simple-NoVaS & $1.16 \times 10^{-7}$ & $1.24 \times 10^{-7}$ & $1.19 \times 10^{-7}$ & $1.21 \times 10^{-7}$ & $1.48 \times 10^{-7}$ \\
Exp-NoVaS & $1.16 \times 10^{-7}$ & $1.24 \times 10^{-7}$ & $1.20 \times 10^{-7}$ & $1.21 \times 10^{-7}$ & $1.49 \times 10^{-7}$ \\
GS-NoVaS & $1.12 \times 10^{-7}$ & $1.22 \times 10^{-7}$ & $1.16 \times 10^{-7}$ & $1.16 \times 10^{-7}$ & $1.45 \times 10^{-7}$ \\
GE-NoVaS & $1.18 \times 10^{-7}$ & $1.27 \times 10^{-7}$ & $1.22 \times 10^{-7}$ & $1.21 \times 10^{-7}$ & $1.50 \times 10^{-7}$ \\
\hline
\end{tabular}


Table 24. MSEs of $L_{2}$ predictions for data generated from smooth transition GARCH(1,1) (ST-GARCH).

\begin{tabular}{cccccc}
\hline Prediction Step & $\mathbf{1}$ & $\mathbf{2}$ & $\mathbf{3}$ & $\mathbf{4}$ & $\mathbf{5}$ \\
\hline Fitting a GARCH & $1.05 \times 10^{-7}$ & $1.13 \times 10^{-7}$ & $9.61 \times 10^{-8}$ & $1.04 \times 10^{-7}$ & $1.25 \times 10^{-7}$ \\
Simple-NoVaS & $1.01 \times 10^{-7}$ & $1.09 \times 10^{-7}$ & $9.96 \times 10^{-8}$ & $1.04 \times 10^{-7}$ & $1.23 \times 10^{-7}$ \\
Exp-NoVaS & $9.93 \times 10^{-8}$ & $1.08 \times 10^{-7}$ & $1.00 \times 10^{-7}$ & $1.03 \times 10^{-7}$ & $1.23 \times 10^{-7}$ \\
GS-NoVaS & $9.84 \times 10^{-8}$ & $1.08 \times 10^{-7}$ & $9.85 \times 10^{-8}$ & $1.00 \times 10^{-7}$ & $1.21 \times 10^{-7}$ \\
GE-NoVaS & $1.00 \times 10^{-7}$ & $1.11 \times 10^{-7}$ & $1.02 \times 10^{-7}$ & $1.02 \times 10^{-7}$ & $1.24 \times 10^{-7}$ \\
\hline
\end{tabular}

Table 25. MADs of $L_{1}$ predictions for data generated from the stochastic volatility model (SV-GARCH).

\begin{tabular}{cccccc}
\hline Prediction Step & $\mathbf{1}$ & $\mathbf{2}$ & $\mathbf{3}$ & $\mathbf{4}$ & $\mathbf{5}$ \\
\hline Fitting a GARCH & $5.35 \times 10^{-5}$ & $5.77 \times 10^{-5}$ & $4.72 \times 10^{-5}$ & $4.28 \times 10^{-5}$ & $3.86 \times 10^{-5}$ \\
Simple-NoVaS & $5.34 \times 10^{-5}$ & $5.69 \times 10^{-5}$ & $4.79 \times 10^{-5}$ & $4.45 \times 10^{-5}$ & $3.78 \times 10^{-5}$ \\
Exp-NoVaS & $5.35 \times 10^{-5}$ & $5.66 \times 10^{-5}$ & $4.74 \times 10^{-5}$ & $4.35 \times 10^{-5}$ & $3.75 \times 10^{-5}$ \\
GS-NoVaS & $5.23 \times 10^{-5}$ & $5.68 \times 10^{-5}$ & $4.74 \times 10^{-5}$ & $4.23 \times 10^{-5}$ & $3.79 \times 10^{-5}$ \\
GE-NoVaS & $5.23 \times 10^{-5}$ & $5.71 \times 10^{-5}$ & $4.79 \times 10^{-5}$ & $4.31 \times 10^{-5}$ & $3.85 \times 10^{-5}$ \\
\hline
\end{tabular}

Table 26. MADs of $L_{2}$ predictions for data generated from the stochastic volatility model (SV-GARCH).

\begin{tabular}{cccccc}
\hline Prediction Step & $\mathbf{1}$ & $\mathbf{2}$ & $\mathbf{3}$ & $\mathbf{4}$ & $\mathbf{5}$ \\
\hline Fitting a GARCH & $5.77 \times 10^{-5}$ & $6.23 \times 10^{-5}$ & $5.56 \times 10^{-5}$ & $5.29 \times 10^{-5}$ & $5.12 \times 10^{-5}$ \\
Simple-NoVaS & $6.02 \times 10^{-5}$ & $6.17 \times 10^{-5}$ & $5.85 \times 10^{-5}$ & $5.99 \times 10^{-5}$ & $5.41 \times 10^{-5}$ \\
Exp-NoVaS & $6.10 \times 10^{-5}$ & $6.19 \times 10^{-5}$ & $5.99 \times 10^{-5}$ & $6.04 \times 10^{-5}$ & $5.68 \times 10^{-5}$ \\
GS-NoVaS & $5.74 \times 10^{-5}$ & $6.29 \times 10^{-5}$ & $6.10 \times 10^{-5}$ & $5.85 \times 10^{-5}$ & $5.69 \times 10^{-5}$ \\
GE-NoVaS & $5.66 \times 10^{-5}$ & $5.96 \times 10^{-5}$ & $5.45 \times 10^{-5}$ & $5.28 \times 10^{-5}$ & $4.77 \times 10^{-5}$ \\
\hline
\end{tabular}

Table 27. MSEs of $L_{1}$ predictions for data generated from the stochastic volatility model (SV-GARCH).

\begin{tabular}{cccccc}
\hline Prediction Step & $\mathbf{1}$ & $\mathbf{2}$ & $\mathbf{3}$ & $\mathbf{4}$ & $\mathbf{5}$ \\
\hline Fitting a GARCH & $1.04 \times 10^{-8}$ & $1.69 \times 10^{-8}$ & $1.08 \times 10^{-8}$ & $7.03 \times 10^{-9}$ & $5.06 \times 10^{-9}$ \\
Simple-NoVaS & $1.02 \times 10^{-8}$ & $1.65 \times 10^{-8}$ & $1.05 \times 10^{-8}$ & $7.06 \times 10^{-9}$ & $4.82 \times 10^{-9}$ \\
Exp-NoVaS & $1.04 \times 10^{-8}$ & $1.65 \times 10^{-8}$ & $1.06 \times 10^{-8}$ & $6.99 \times 10^{-9}$ & $4.78 \times 10^{-9}$ \\
GS-NoVaS & $1.00 \times 10^{-8}$ & $1.66 \times 10^{-8}$ & $1.04 \times 10^{-8}$ & $6.92 \times 10^{-9}$ & $4.80 \times 10^{-9}$ \\
GE-NoVaS & $1.03 \times 10^{-8}$ & $1.70 \times 10^{-8}$ & $1.08 \times 10^{-8}$ & $6.94 \times 10^{-9}$ & $5.04 \times 10^{-9}$ \\
\hline
\end{tabular}

Table 28. MSEs of $L_{2}$ predictions for data generated from the stochastic volatility model (SV-GARCH).

\begin{tabular}{cccccc}
\hline Prediction Step & $\mathbf{1}$ & $\mathbf{2}$ & $\mathbf{3}$ & $\mathbf{4}$ & $\mathbf{5}$ \\
\hline Fitting a GARCH & $8.99 \times 10^{-9}$ & $1.51 \times 10^{-8}$ & $1.02 \times 10^{-8}$ & $6.76 \times 10^{-9}$ & $5.29 \times 10^{-9}$ \\
Simple-NoVaS & $8.70 \times 10^{-9}$ & $1.42 \times 10^{-8}$ & $9.99 \times 10^{-9}$ & $7.62 \times 10^{-9}$ & $5.49 \times 10^{-9}$ \\
Exp-NoVaS & $8.82 \times 10^{-9}$ & $1.39 \times 10^{-8}$ & $1.00 \times 10^{-8}$ & $7.41 \times 10^{-9}$ & $5.51 \times 10^{-9}$ \\
GS-NoVaS & $8.10 \times 10^{-9}$ & $1.42 \times 10^{-8}$ & $1.02 \times 10^{-8}$ & $6.92 \times 10^{-9}$ & $5.32 \times 10^{-9}$ \\
GE-NoVaS & $8.37 \times 10^{-9}$ & $1.46 \times 10^{-8}$ & $1.00 \times 10^{-8}$ & $6.62 \times 10^{-9}$ & $5.27 \times 10^{-9}$ \\
\hline
\end{tabular}

As regards prediction intervals, the simulation results are summarized in Tables 29-35. Generally, the conclusions were similar to those of the point predictions. Furthermore, NoVaS methods gave more accurate coverage than $\operatorname{GARCH}(1,1)$ in the $L_{1}$ sense prediction for all seven data generating processes. In the $L_{2}$ sense, when the data were generated from a standard GARCH $(1,1)$ with normal errors, GARCH $(1,1)$ gave as good coverage as NoVaS. When we used other models to generate data, for example, GARCH $(1,1)$ with $t$ distributed errors or MS-GARCH $(1,1)$ or ST-GARCH(1,1) etc., GARCH(1,1) performed very poorly, while NoVaS methods were still performing well. 
Table 29. Interval predictions for data generated from $\operatorname{GARCH}(1,1)$ with $\omega=0.00001, \alpha=0.8895, \theta=$ 0.10 , and $\epsilon \sim$ i.i.d $N(0,1)$. CVR, average coverage level; LEN, average length.

\begin{tabular}{|c|c|c|c|c|c|c|c|}
\hline \multicolumn{4}{|c|}{ L2 } & \multicolumn{4}{|c|}{ L1 } \\
\hline \multicolumn{4}{|c|}{ GARCH $(1,1)$} & \multicolumn{4}{|c|}{ GARCH $(1,1)$} \\
\hline STEPS & CVR & LEN & ST.ERR & STEPS & CVR & LEN & ST.ERR \\
\hline 1 & 0.714 & $1.28 \times 10^{-2}$ & $1.77 \times 10^{-2}$ & 1 & 0.744 & $1.01 \times 10^{-2}$ & $1.55 \times 10^{-2}$ \\
\hline 2 & 0.746 & $1.28 \times 10^{-2}$ & $1.79 \times 10^{-2}$ & 2 & 0.734 & $1.13 \times 10^{-2}$ & $1.83 \times 10^{-2}$ \\
\hline 3 & 0.746 & $1.37 \times 10^{-2}$ & $1.87 \times 10^{-2}$ & 3 & 0.768 & $1.19 \times 10^{-2}$ & $1.95 \times 10^{-2}$ \\
\hline 4 & 0.766 & $1.18 \times 10^{-2}$ & $1.59 \times 10^{-2}$ & 4 & 0.734 & $1.20 \times 10^{-2}$ & $1.99 \times 10^{-2}$ \\
\hline 5 & 0.786 & $1.28 \times 10^{-2}$ & $1.75 \times 10^{-2}$ & 5 & 0.744 & $1.28 \times 10^{-2}$ & $2.04 \times 10^{-2}$ \\
\hline \multicolumn{4}{|c|}{ EXP-NoVaS } & \multicolumn{4}{|c|}{ EXP-NoVaS } \\
\hline STEPS & CVR & LEN & ST.ERR & STEPS & CVR & LEN & ST.ERR \\
\hline 1 & 0.958 & $2.00 \times 10^{-2}$ & $1.35 \times 10^{-2}$ & 1 & 0.936 & $1.99 \times 10^{-2}$ & $1.39 \times 10^{-2}$ \\
\hline 2 & 0.952 & $2.07 \times 10^{-2}$ & $1.44 \times 10^{-2}$ & 2 & 0.936 & $2.19 \times 10^{-2}$ & $1.52 \times 10^{-2}$ \\
\hline 3 & 0.952 & $1.98 \times 10^{-2}$ & $1.27 \times 10^{-2}$ & 3 & 0.944 & $1.94 \times 10^{-2}$ & $1.27 \times 10^{-2}$ \\
\hline 4 & 0.946 & $2.17 \times 10^{-2}$ & $1.43 \times 10^{-2}$ & 4 & 0.944 & $2.08 \times 10^{-2}$ & $1.28 \times 10^{-2}$ \\
\hline 5 & 0.950 & $2.19 \times 10^{-2}$ & $1.32 \times 10^{-2}$ & 5 & 0.936 & $2.19 \times 10^{-2}$ & $1.51 \times 10^{-2}$ \\
\hline \multicolumn{4}{|c|}{ Simple-NoVaS } & \multicolumn{4}{|c|}{ Simple-NoVaS } \\
\hline TEPS & CVR & LEN & ST.ERR & ГEPS & CVR & LEN & ST.ERR \\
\hline 1 & 0.946 & $1.98 \times 10^{-2}$ & $1.35 \times 10^{-2}$ & 1 & 0.934 & $1.84 \times 10^{-2}$ & $1.32 \times 10^{-2}$ \\
\hline 2 & 0.942 & $1.78 \times 10^{-2}$ & $1.41 \times 10^{-2}$ & 2 & 0.946 & $1.91 \times 10^{-2}$ & $1.49 \times 10^{-2}$ \\
\hline 3 & 0.946 & $1.92 \times 10^{-2}$ & $1.35 \times 10^{-2}$ & 3 & 0.936 & $2.04 \times 10^{-2}$ & $1.47 \times 10^{-2}$ \\
\hline 4 & 0.946 & $2.07 \times 10^{-2}$ & $1.34 \times 10^{-2}$ & 4 & 0.964 & $1.93 \times 10^{-2}$ & $1.40 \times 10^{-2}$ \\
\hline 5 & 0.956 & $2.21 \times 10^{-2}$ & $1.43 \times 10^{-2}$ & 5 & 0.954 & $2.07 \times 10^{-2}$ & $1.45 \times 10^{-2}$ \\
\hline \multicolumn{4}{|c|}{ GS-NoVaS } & \multicolumn{4}{|c|}{ GS-NoVaS } \\
\hline TEPS & CVR & LEN & ST.ERR & STEPS & CVR & LEN & ST.ERR \\
\hline 1 & 0.948 & $2.05 \times 10^{-2}$ & $1.39 \times 10^{-2}$ & 1 & 0.95 & $1.61 \times 10^{-2}$ & $1.21 \times 10^{-2}$ \\
\hline 2 & 0.942 & $1.78 \times 10^{-2}$ & $1.41 \times 10^{-2}$ & 2 & 0.94 & $2.07 \times 10^{-2}$ & $1.47 \times 10^{-2}$ \\
\hline 3 & 0.952 & $1.93 \times 10^{-2}$ & $1.43 \times 10^{-2}$ & 3 & 0.936 & $1.62 \times 10^{-2}$ & $1.25 \times 10^{-2}$ \\
\hline 4 & 0.948 & $2.14 \times 10^{-2}$ & $1.30 \times 10^{-2}$ & 4 & 0.936 & $1.70 \times 10^{-2}$ & $1.42 \times 10^{-2}$ \\
\hline 5 & 0.954 & $2.26 \times 10^{-2}$ & $1.40 \times 10^{-2}$ & 5 & 0.94 & $1.78 \times 10^{-2}$ & $1.32 \times 10^{-2}$ \\
\hline \multicolumn{4}{|c|}{ GE-NoVaS } & \multicolumn{4}{|c|}{ GE-NoVaS } \\
\hline STEPS & CVR & LEN & ST.ERR & STEPS & CVR & LEN & ST.ERR \\
\hline 1 & 0.948 & $1.73 \times 10^{-2}$ & $1.14 \times 10^{-2}$ & 1 & 0.958 & $1.98 \times 10^{-2}$ & $1.13 \times 10^{-2}$ \\
\hline 2 & 0.948 & $1.66 \times 10^{-2}$ & $1.05 \times 10^{-2}$ & 2 & 0.942 & $1.68 \times 10^{-2}$ & $1.16 \times 10^{-2}$ \\
\hline 3 & 0.952 & $1.98 \times 10^{-2}$ & $1.27 \times 10^{-2}$ & 3 & 0.944 & $1.94 \times 10^{-2}$ & $1.27 \times 10^{-2}$ \\
\hline 4 & 0.946 & $2.17 \times 10^{-2}$ & $1.43 \times 10^{-2}$ & 4 & 0.944 & $2.08 \times 10^{-2}$ & $1.28 \times 10^{-2}$ \\
\hline 5 & 0.950 & $2.19 \times 10^{-2}$ & $1.32 \times 10^{-2}$ & 5 & 0.942 & $2.32 \times 10^{-2}$ & $1.13 \times 10^{-2}$ \\
\hline
\end{tabular}

Table 30. Interval predictions for data generated from $\operatorname{GARCH}(1,1)$ with $\omega=0.00001, \alpha=0.73, \theta=$ 0.10 , and $\epsilon \sim$ i.i.d $N(0,1)$.

\begin{tabular}{|c|c|c|c|c|c|c|c|}
\hline \multicolumn{4}{|c|}{ L2 } & \multicolumn{4}{|c|}{ L1 } \\
\hline \multicolumn{4}{|c|}{ GARCH(1,1) } & \multicolumn{4}{|c|}{ GARCH $(1,1)$} \\
\hline STEPS & CVR & LEN & ST.ERR & STEPS & CVR & LEN & ST.ERR \\
\hline 1 & 0.948 & $4.39 \times 10^{-3}$ & $2.11 \times 10^{-3}$ & 1 & 0.92 & $3.76 \times 10^{-3}$ & $3.18 \times 10^{-3}$ \\
\hline 2 & 0.940 & $4.53 \times 10^{-3}$ & $2.23 \times 10^{-3}$ & 2 & 0.936 & $5.49 \times 10^{-3}$ & $5.21 \times 10^{-3}$ \\
\hline 3 & 0.950 & $4.47 \times 10^{-3}$ & $2.74 \times 10^{-3}$ & 3 & 0.938 & $5.99 \times 10^{-3}$ & $5.52 \times 10^{-3}$ \\
\hline 4 & 0.952 & $4.02 \times 10^{-3}$ & $1.89 \times 10^{-3}$ & 4 & 0.922 & $7.16 \times 10^{-3}$ & $6.31 \times 10^{-3}$ \\
\hline 5 & 0.934 & $3.77 \times 10^{-3}$ & $2.74 \times 10^{-3}$ & 5 & 0.92 & $4.57 \times 10^{-3}$ & $4.21 \times 10^{-3}$ \\
\hline
\end{tabular}


Table 30. Cont.

\begin{tabular}{|c|c|c|c|c|c|c|c|}
\hline \multicolumn{4}{|c|}{ L2 } & \multicolumn{4}{|c|}{ L1 } \\
\hline \multicolumn{4}{|c|}{ EXP-NoVaS } & \multicolumn{4}{|c|}{ EXP-NoVaS } \\
\hline STEPS & CVR & LEN & ST.ERR & STEPS & CVR & LEN & ST.ERR \\
\hline 1 & 0.954 & $4.46 \times 10^{-3}$ & $2.69 \times 10^{-3}$ & 1 & 0.954 & $4.74 \times 10^{-3}$ & $2.29 \times 10^{-3}$ \\
\hline 2 & 0.972 & $4.40 \times 10^{-3}$ & $2.55 \times 10^{-3}$ & 2 & 0.95 & $4.62 \times 10^{-3}$ & $2.19 \times 10^{-3}$ \\
\hline 3 & 0.938 & $4.17 \times 10^{-3}$ & $2.59 \times 10^{-3}$ & 3 & 0.95 & $4.64 \times 10^{-3}$ & $2.12 \times 10^{-3}$ \\
\hline 4 & 0.958 & $4.62 \times 10^{-3}$ & $2.59 \times 10^{-3}$ & 4 & 0.948 & $4.58 \times 10^{-3}$ & $2.12 \times 10^{-3}$ \\
\hline 5 & 0.950 & $4.58 \times 10^{-3}$ & $2.49 \times 10^{-3}$ & 5 & 0.942 & $4.45 \times 10^{-3}$ & $1.88 \times 10^{-3}$ \\
\hline \multicolumn{4}{|c|}{ Simple-NoVaS } & \multicolumn{4}{|c|}{ Simple-NoVaS } \\
\hline TEPS & CVR & LEN & ST.E & STEPS & CVR & LEN & ST.ERR \\
\hline 1 & 0.960 & $4.54 \times 10^{-3}$ & $2.50 \times 10^{-3}$ & 1 & 0.946 & $4.28 \times 10^{-3}$ & $2.45 \times 10^{-3}$ \\
\hline 2 & 0.958 & $4.27 \times 10^{-3}$ & $2.97 \times 10^{-3}$ & 2 & 0.95 & $4.26 \times 10^{-3}$ & $2.33 \times 10^{-3}$ \\
\hline 3 & 0.968 & $4.63 \times 10^{-3}$ & $2.87 \times 10^{-3}$ & 3 & 0.952 & $4.21 \times 10^{-3}$ & $2.62 \times 10^{-3}$ \\
\hline 4 & 0.960 & $4.73 \times 10^{-3}$ & $2.85 \times 10^{-3}$ & 4 & 0.954 & $4.25 \times 10^{-3}$ & $2.55 \times 10^{-3}$ \\
\hline 5 & 0.948 & $4.15 \times 10^{-3}$ & $2.93 \times 10^{-3}$ & 5 & 0.948 & $4.19 \times 10^{-3}$ & $2.32 \times 10^{-3}$ \\
\hline \multicolumn{4}{|c|}{ GS-NoVaS } & \multicolumn{4}{|c|}{ GS-NoVaS } \\
\hline TEP & CVR & LEN & ST.ERR & STEPS & CVR & LEN & ST.ERR \\
\hline 1 & 0.949 & $4.37 \times 10^{-3}$ & $2.53 \times 10^{-3}$ & 1 & 0.946 & $4.26 \times 10^{-3}$ & $2.37 \times 10^{-3}$ \\
\hline 2 & 0.948 & $4.63 \times 10^{-2}$ & $2.78 \times 10^{-3}$ & 2 & 0.95 & $4.26 \times 10^{-3}$ & $2.33 \times 10^{-3}$ \\
\hline 3 & 0.938 & $4.17 \times 10^{-3}$ & $2.59 \times 10^{-3}$ & 3 & 0.95 & $4.22 \times 10^{-3}$ & $2.42 \times 10^{-3}$ \\
\hline 4 & 0.945 & $3.76 \times 10^{-3}$ & $2.71 \times 10^{-3}$ & 4 & 0.948 & $4.20 \times 10^{-3}$ & $1.91 \times 10^{-3}$ \\
\hline 5 & 0.950 & $4.58 \times 10^{-3}$ & $2.49 \times 10^{-3}$ & 5 & 0.948 & $4.19 \times 10^{-3}$ & $2.32 \times 10^{-3}$ \\
\hline \multicolumn{4}{|c|}{ GE-NoVaS } & \multicolumn{4}{|c|}{ GE-NoVaS } \\
\hline STEPS & CVR & LEN & ST.ERR & STEPS & CVR & LEN & ST.ERR \\
\hline 1 & 0.946 & $4.92 \times 10^{-3}$ & $2.57 \times 10^{-3}$ & 1 & 0.96 & $5.37 \times 10^{-3}$ & $2.00 \times 10^{-3}$ \\
\hline 2 & 0.946 & $4.68 \times 10^{-3}$ & $2.38 \times 10^{-3}$ & 2 & 0.948 & $5.13 \times 10^{-3}$ & $3.34 \times 10^{-3}$ \\
\hline 3 & 0.958 & $4.39 \times 10^{-3}$ & $2.35 \times 10^{-3}$ & 3 & 0.952 & $4.03 \times 10^{-3}$ & $2.05 \times 10^{-3}$ \\
\hline 4 & 0.954 & $4.30 \times 10^{-3}$ & $2.03 \times 10^{-3}$ & 4 & 0.95 & $4.80 \times 10^{-3}$ & $2.03 \times 10^{-3}$ \\
\hline 5 & 0.948 & $4.15 \times 10^{-3}$ & $2.93 \times 10^{-3}$ & 5 & 0.944 & $4.42 \times 10^{-3}$ & $2.78 \times 10^{-3}$ \\
\hline
\end{tabular}

Table 31. Results of interval predictions for data generated from $\operatorname{GARCH}(1,1)$ with $\omega=0.00001, \alpha=$ $0.73, \theta=0.10$, and $\epsilon \sim$ i.i.d $t_{5}$.

\begin{tabular}{cccccccc}
\hline \multicolumn{3}{c}{ L2 } & \multicolumn{5}{c}{ L1 } \\
\hline \multicolumn{3}{c}{ GARCH(1,1) } \\
\hline STEPS & CVR & LEN & ST.ERR & STEPS & CVR & LEN & ST.ERR \\
1 & 0.924 & $4.17 \times 10^{-3}$ & $3.18 \times 10^{-3}$ & 1 & 0.936 & $2.35 \times 10^{-3}$ & $8.60 \times 10^{-3}$ \\
2 & 0.931 & $3.75 \times 10^{-3}$ & $2.57 \times 10^{-3}$ & 2 & 0.928 & $2.15 \times 10^{-3}$ & $7.50 \times 10^{-3}$ \\
3 & 0.922 & $4.47 \times 10^{-3}$ & $2.24 \times 10^{-3}$ & 3 & 0.92 & $2.37 \times 10^{-3}$ & $8.52 \times 10^{-3}$ \\
4 & 0.925 & $4.02 \times 10^{-3}$ & $2.63 \times 10^{-3}$ & 4 & 0.938 & $2.92 \times 10^{-3}$ & $6.95 \times 10^{-3}$ \\
5 & 0.922 & $4.56 \times 10^{-3}$ & $2.79 \times 10^{-3}$ & 5 & 0.92 & $2.79 \times 10^{-3}$ & $8.20 \times 10^{-3}$ \\
\hline & & & \multicolumn{5}{c}{ EXP-NoVaS } \\
\hline STEPS & CVR & LEN & ST.ERR & STEPS & CVR & LEN \\
1 & 0.945 & $4.46 \times 10^{-3}$ & $2.66 \times 10^{-3}$ & 1 & 0.95 & $3.38 \times 10^{-3}$ & $2.95 \times 10^{-3}$ \\
2 & 0.942 & $4.25 \times 10^{-3}$ & $2.72 \times 10^{-3}$ & 2 & 0.958 & $3.80 \times 10^{-3}$ & $2.72 \times 10^{-3}$ \\
3 & 0.943 & $4.54 \times 10^{-3}$ & $2.77 \times 10^{-3}$ & 3 & 0.946 & $3.75 \times 10^{-3}$ & $2.40 \times 10^{-3}$ \\
4 & 0.949 & $4.94 \times 10^{-3}$ & $2.72 \times 10^{-3}$ & 4 & 0.952 & $3.76 \times 10^{-3}$ & $2.43 \times 10^{-3}$ \\
5 & 0.954 & $4.72 \times 10^{-3}$ & $3.08 \times 10^{-3}$ & 5 & 0.946 & $3.40 \times 10^{-3}$ & $2.84 \times 10^{-3}$ \\
\hline
\end{tabular}


Table 31. Cont.

\begin{tabular}{|c|c|c|c|c|c|c|c|}
\hline \multicolumn{4}{|c|}{ L2 } & \multicolumn{4}{|c|}{ L1 } \\
\hline \multicolumn{4}{|c|}{ Simple-NoVaS } & \multicolumn{4}{|c|}{ Simple-NoVaS } \\
\hline STEPS & CVR & LEN & ST.ERR & STEPS & CVR & LEN & ST.ERR \\
\hline 1 & 0.950 & $4.82 \times 10^{-3}$ & $2.35 \times 10^{-3}$ & 1 & 0.953 & $4.01 \times 10^{-3}$ & $3.07 \times 10^{-3}$ \\
\hline 2 & 0.963 & $4.86 \times 10^{-3}$ & $3.26 \times 10^{-3}$ & 2 & 0.942 & $3.58 \times 10^{-3}$ & $2.73 \times 10^{-3}$ \\
\hline 3 & 0.966 & $4.85 \times 10^{-3}$ & $2.82 \times 10^{-3}$ & 3 & 0.952 & $3.28 \times 10^{-3}$ & $2.48 \times 10^{-3}$ \\
\hline 4 & 0.954 & $5.04 \times 10^{-3}$ & $3.05 \times 10^{-3}$ & 4 & 0.952 & $3.51 \times 10^{-3}$ & $2.54 \times 10^{-3}$ \\
\hline 5 & 0.944 & $4.36 \times 10^{-3}$ & $2.51 \times 10^{-3}$ & 5 & 0.944 & $4.20 \times 10^{-3}$ & $3.03 \times 10^{-3}$ \\
\hline \multicolumn{4}{|c|}{ GS-NoVaS } & \multicolumn{4}{|c|}{ GS-NoVaS } \\
\hline STEPS & CVR & LEN & ST.ERR & STEPS & CVR & LEN & ST.ERR \\
\hline 1 & 0.950 & $4.82 \times 10^{-3}$ & $2.35 \times 10^{-3}$ & 1 & 0.948 & $3.64 \times 10^{-3}$ & $4.49 \times 10^{-3}$ \\
\hline 2 & 0.954 & $4.47 \times 10^{-3}$ & $2.93 \times 10^{-3}$ & 2 & 0.956 & $3.22 \times 10^{-3}$ & $5.92 \times 10^{-3}$ \\
\hline 3 & 0.952 & $4.69 \times 10^{-3}$ & $3.03 \times 10^{-3}$ & 3 & 0.946 & $3.31 \times 10^{-3}$ & $4.08 \times 10^{-3}$ \\
\hline 4 & 0.950 & $4.57 \times 10^{-3}$ & $2.99 \times 10^{-3}$ & 4 & 0.948 & $3.23 \times 10^{-3}$ & $4.52 \times 10^{-3}$ \\
\hline 5 & 0.954 & $4.50 \times 10^{-3}$ & $3.06 \times 10^{-3}$ & 5 & 0.95 & $3.62 \times 10^{-3}$ & $4.54 \times 10^{-3}$ \\
\hline \multicolumn{4}{|c|}{ GE-NoVaS } & \multicolumn{4}{|c|}{ GE-NoVaS } \\
\hline STEPS & CVR & LEN & ST.ERR & STEPS & CVR & LEN & ST.ERR \\
\hline 1 & 0.950 & $4.90 \times 10^{-3}$ & $2.57 \times 10^{-3}$ & 1 & 0.954 & $3.65 \times 10^{-3}$ & $2.78 \times 10^{-3}$ \\
\hline 2 & 0.946 & $4.36 \times 10^{-3}$ & $2.93 \times 10^{-3}$ & 2 & 0.954 & $3.86 \times 10^{-3}$ & $2.84 \times 10^{-3}$ \\
\hline 3 & 0.948 & $4.48 \times 10^{-3}$ & $2.82 \times 10^{-3}$ & 3 & 0.946 & $3.64 \times 10^{-3}$ & $2.81 \times 10^{-3}$ \\
\hline 4 & 0.952 & $4.58 \times 10^{-3}$ & $2.78 \times 10^{-3}$ & 4 & 0.95 & $3.53 \times 10^{-3}$ & $2.89 \times 10^{-3}$ \\
\hline 5 & 0.952 & $4.53 \times 10^{-3}$ & $2.94 \times 10^{-3}$ & 5 & 0.966 & $6.38 \times 10^{-3}$ & $3.92 \times 10^{-3}$ \\
\hline
\end{tabular}

Table 32. Results of interval predictions for data generated from TV-GARCH(1,1).

\begin{tabular}{|c|c|c|c|c|c|c|c|}
\hline \multicolumn{4}{|c|}{ L2 } & \multicolumn{4}{|c|}{ L1 } \\
\hline \multicolumn{4}{|c|}{ GARCH $(1,1)$} & \multicolumn{4}{|c|}{ GARCH(1,1) } \\
\hline STEPS & CVR & LEN & ST.ERR & STEPS & CVR & LEN & ST.ERR \\
\hline 1 & 0.850 & $1.57 \times 10^{-3}$ & $1.07 \times 10^{-2}$ & 1 & 0.726 & $1.12 \times 10^{-3}$ & $4.97 \times 10^{-3}$ \\
\hline 2 & 0.848 & $1.20 \times 10^{-3}$ & $1.60 \times 10^{-3}$ & 2 & 0.716 & $1.32 \times 10^{-3}$ & $5.88 \times 10^{-3}$ \\
\hline 3 & 0.858 & $1.84 \times 10^{-3}$ & $2.77 \times 10^{-3}$ & 3 & 0.718 & $8.87 \times 10^{-3}$ & $3.90 \times 10^{-3}$ \\
\hline 4 & 0.844 & $2.32 \times 10^{-3}$ & $2.02 \times 10^{-3}$ & 4 & 0.716 & $9.78 \times 10^{-3}$ & $4.39 \times 10^{-3}$ \\
\hline 5 & 0.856 & $2.29 \times 10^{-3}$ & $1.68 \times 10^{-3}$ & 5 & 0.72 & $1.24 \times 10^{-3}$ & $5.77 \times 10^{-3}$ \\
\hline \multicolumn{4}{|c|}{ EXP-NoVaS } & \multicolumn{4}{|c|}{ EXP-NoVaS } \\
\hline STEPS & CVR & LEN & ST.ERR & STEPS & CVR & LEN & ST.ERR \\
\hline 1 & 0.948 & $2.40 \times 10^{-3}$ & $2.20 \times 10^{-3}$ & 1 & 0.952 & $2.76 \times 10^{-3}$ & $2.45 \times 10^{-3}$ \\
\hline 2 & 0.950 & $2.23 \times 10^{-3}$ & $2.17 \times 10^{-3}$ & 2 & 0.952 & $2.74 \times 10^{-3}$ & $2.50 \times 10^{-3}$ \\
\hline 3 & 0.952 & $2.93 \times 10^{-3}$ & $2.15 \times 10^{-3}$ & 3 & 0.95 & $2.69 \times 10^{-3}$ & $2.62 \times 10^{-3}$ \\
\hline 4 & 0.954 & $3.02 \times 10^{-3}$ & $2.18 \times 10^{-3}$ & 4 & 0.942 & $2.78 \times 10^{-3}$ & $2.65 \times 10^{-3}$ \\
\hline 5 & 0.950 & $2.86 \times 10^{-3}$ & $2.12 \times 10^{-3}$ & 5 & 0.948 & $2.82 \times 10^{-3}$ & $2.61 \times 10^{-3}$ \\
\hline \multicolumn{4}{|c|}{ Simple-NoVaS } & \multicolumn{4}{|c|}{ Simple-NoVaS } \\
\hline STEPS & CVR & LEN & ST.ERR & STEPS & CVR & LEN & ST.ERR \\
\hline 1 & 0.950 & $2.32 \times 10^{-3}$ & $2.37 \times 10^{-3}$ & 1 & 0.942 & $2.20 \times 10^{-3}$ & $2.30 \times 10^{-3}$ \\
\hline 2 & 0.956 & $2.20 \times 10^{-3}$ & $2.36 \times 10^{-3}$ & 2 & 0.956 & $2.82 \times 10^{-3}$ & $2.45 \times 10^{-3}$ \\
\hline 3 & 0.960 & $2.88 \times 10^{-3}$ & $2.15 \times 10^{-3}$ & 3 & 0.948 & $2.60 \times 10^{-3}$ & $2.50 \times 10^{-3}$ \\
\hline 4 & 0.952 & $2.50 \times 10^{-3}$ & $2.27 \times 10^{-3}$ & 4 & 0.946 & $2.79 \times 10^{-3}$ & $2.36 \times 10^{-3}$ \\
\hline 5 & 0.954 & $2.80 \times 10^{-3}$ & $2.01 \times 10^{-3}$ & 5 & 0.946 & $2.47 \times 10^{-3}$ & $2.62 \times 10^{-3}$ \\
\hline
\end{tabular}


Table 32. Cont.

\begin{tabular}{cccccccc}
\hline \multicolumn{3}{c}{ L2 } & \multicolumn{5}{c}{ L1 } \\
\hline & \multicolumn{5}{c}{ GS-NoVaS } & & GS-NoVaS \\
\hline STEPS & CVR & LEN & ST.ERR & STEPS & CVR & LEN & ST.ERR \\
1 & 0.950 & $2.32 \times 10^{-3}$ & $2.37 \times 10^{-3}$ & 1 & 0.942 & $2.20 \times 10^{-3}$ & $2.30 \times 10^{-3}$ \\
2 & 0.950 & $2.52 \times 10^{-3}$ & $2.57 \times 10^{-3}$ & 2 & 0.948 & $2.57 \times 10^{-3}$ & $2.33 \times 10^{-3}$ \\
3 & 0.952 & $2.59 \times 10^{-3}$ & $2.53 \times 10^{-3}$ & 3 & 0.952 & $2.52 \times 10^{-3}$ & $2.18 \times 10^{-3}$ \\
4 & 0.950 & $2.37 \times 10^{-3}$ & $2.05 \times 10^{-3}$ & 4 & 0.946 & $2.97 \times 10^{-3}$ & $2.22 \times 10^{-3}$ \\
5 & 0.950 & $2.62 \times 10^{-3}$ & $2.12 \times 10^{-3}$ & 5 & 0.95 & $2.64 \times 10^{-3}$ & $2.21 \times 10^{-3}$ \\
\hline & & GE-NoVaS & & & & GE-NoVaS & \\
\hline STEPS & CVR & LEN & ST.ERR & STEPS & CVR & LEN & ST.ERR \\
1 & 0.948 & $2.40 \times 10^{-3}$ & $2.20 \times 10^{-3}$ & 1 & 0.948 & $2.77 \times 10^{-3}$ & $2.66 \times 10^{-3}$ \\
2 & 0.950 & $2.23 \times 10^{-3}$ & $2.17 \times 10^{-3}$ & 2 & 0.952 & $2.71 \times 10^{-3}$ & $2.83 \times 10^{-3}$ \\
3 & 0.942 & $2.47 \times 10^{-3}$ & $2.25 \times 10^{-3}$ & 3 & 0.95 & $2.53 \times 10^{-3}$ & $2.50 \times 10^{-3}$ \\
4 & 0.948 & $2.44 \times 10^{-3}$ & $2.17 \times 10^{-3}$ & 4 & 0.95 & $2.75 \times 10^{-3}$ & $2.49 \times 10^{-3}$ \\
5 & 0.949 & $2.29 \times 10^{-3}$ & $2.12 \times 10^{-3}$ & 5 & 0.954 & $2.64 \times 10^{-3}$ & $2.47 \times 10^{-3}$ \\
\hline
\end{tabular}

Table 33. Results of interval predictions for data generated from MS-GARCH(1,1).

\begin{tabular}{|c|c|c|c|c|c|c|c|}
\hline \multicolumn{4}{|c|}{ L2 } & \multicolumn{4}{|c|}{ L1 } \\
\hline \multicolumn{4}{|c|}{ GARCH(1,1) } & \multicolumn{4}{|c|}{ GARCH $(1,1)$} \\
\hline STEPS & CVR & LEN & ST.ERR & STEPS & CVR & LEN & ST.ERR \\
\hline 1 & 0.868 & $3.32 \times 10^{-2}$ & $1.78 \times 10^{-2}$ & 1 & 0.856 & $3.27 \times 10^{-2}$ & $1.25 \times 10^{-2}$ \\
\hline 2 & 0.872 & $3.42 \times 10^{-2}$ & $1.50 \times 10^{-2}$ & 2 & 0.89 & $3.04 \times 10^{-2}$ & $1.10 \times 10^{-2}$ \\
\hline 3 & 0.868 & $3.58 \times 10^{-2}$ & $1.62 \times 10^{-2}$ & 3 & 0.882 & $3.10 \times 10^{-2}$ & $1.07 \times 10^{-2}$ \\
\hline 4 & 0.858 & $3.67 \times 10^{-2}$ & $1.86 \times 10^{-2}$ & 4 & 0.886 & $3.09 \times 10^{-2}$ & $1.12 \times 10^{-2}$ \\
\hline 5 & 0.87 & $3.60 \times 10^{-2}$ & $2.10 \times 10^{-2}$ & 5 & 0.908 & $7.66 \times 10^{-3}$ & $1.28 \times 10^{-2}$ \\
\hline \multicolumn{4}{|c|}{ EXP-NoVaS } & \multicolumn{4}{|c|}{ EXP-NoVaS } \\
\hline TEPS & CVR & LEN & ST.ERR & STEPS & CVR & LEN & ST.ERR \\
\hline 1 & 0.946 & $4.14 \times 10^{-2}$ & $2.18 \times 10^{-2}$ & 1 & 0.952 & $3.97 \times 10^{-2}$ & $2.67 \times 10^{-2}$ \\
\hline 2 & 0.948 & $4.02 \times 10^{-2}$ & $2.26 \times 10^{-2}$ & 2 & 0.944 & $4.22 \times 10^{-2}$ & $2.80 \times 10^{-2}$ \\
\hline 3 & 0.96 & $4.78 \times 10^{-2}$ & $2.19 \times 10^{-2}$ & 3 & 0.958 & $3.99 \times 10^{-2}$ & $2.74 \times 10^{-2}$ \\
\hline 4 & 0.958 & $4.16 \times 10^{-2}$ & $2.06 \times 10^{-2}$ & 4 & 0.938 & $3.86 \times 10^{-2}$ & $2.80 \times 10^{-2}$ \\
\hline 5 & 0.956 & $4.27 \times 10^{-2}$ & $2.07 \times 10^{-2}$ & 5 & 0.944 & $4.20 \times 10^{-2}$ & $2.97 \times 10^{-2}$ \\
\hline \multicolumn{4}{|c|}{ Simple-NoVaS } & \multicolumn{4}{|c|}{ Simple-NoVaS } \\
\hline TEPS & CVR & LEN & ST.ERR & STEPS & CVR & LEN & ST.ERR \\
\hline 1 & 0.954 & $4.21 \times 10^{-2}$ & $2.87 \times 10^{-2}$ & 1 & 0.958 & $3.05 \times 10^{-2}$ & $2.03 \times 10^{-2}$ \\
\hline 2 & 0.948 & $3.98 \times 10^{-2}$ & $2.81 \times 10^{-2}$ & 2 & 0.936 & $3.08 \times 10^{-2}$ & $2.26 \times 10^{-2}$ \\
\hline 3 & 0.94 & $4.47 \times 10^{-2}$ & $2.91 \times 10^{-2}$ & 3 & 0.936 & $3.45 \times 10^{-2}$ & $2.13 \times 10^{-2}$ \\
\hline 4 & 0.948 & $4.26 \times 10^{-2}$ & $2.80 \times 10^{-2}$ & 4 & 0.94 & $3.42 \times 10^{-2}$ & $2.28 \times 10^{-2}$ \\
\hline 5 & 0.946 & $4.32 \times 10^{-2}$ & $2.93 \times 10^{-2}$ & 5 & 0.938 & $3.52 \times 10^{-2}$ & $2.16 \times 10^{-2}$ \\
\hline \multicolumn{4}{|c|}{ GS-NoVaS } & \multicolumn{4}{|c|}{ GS-NoVaS } \\
\hline TEPS & CVR & LEN & ST.ERR & STEPS & CVR & LEN & ST.ERR \\
\hline 1 & 0.954 & $4.21 \times 10^{-2}$ & $2.87 \times 10^{-2}$ & 1 & 0.948 & $3.21 \times 10^{-2}$ & $2.16 \times 10^{-2}$ \\
\hline 2 & 0.948 & $3.98 \times 10^{-2}$ & $2.81 \times 10^{-2}$ & 2 & 0.946 & $3.26 \times 10^{-2}$ & $2.18 \times 10^{-2}$ \\
\hline 3 & 0.942 & $4.75 \times 10^{-2}$ & $2.84 \times 10^{-2}$ & 3 & 0.948 & $3.46 \times 10^{-2}$ & $2.28 \times 10^{-2}$ \\
\hline 4 & 0.948 & $4.26 \times 10^{-2}$ & $2.80 \times 10^{-2}$ & 4 & 0.952 & $3.17 \times 10^{-2}$ & $2.21 \times 10^{-2}$ \\
\hline 5 & 0.946 & $4.32 \times 10^{-2}$ & $2.93 \times 10^{-2}$ & 5 & 0.946 & $3.22 \times 10^{-2}$ & $2.02 \times 10^{-2}$ \\
\hline
\end{tabular}


Table 33. Cont.

\begin{tabular}{|c|c|c|c|c|c|c|c|}
\hline \multicolumn{4}{|c|}{ L2 } & \multicolumn{4}{|c|}{ L1 } \\
\hline \multicolumn{4}{|c|}{ GE-NoVaS } & \multicolumn{4}{|c|}{ GE-NoVaS } \\
\hline STEPS & CVR & LEN & ST.ERR & STEPS & CVR & LEN & ST.ERR \\
\hline 1 & 0.954 & $4.30 \times 10^{-2}$ & $2.08 \times 10^{-2}$ & 1 & 0.948 & $3.40 \times 10^{-2}$ & $2.19 \times 10^{-2}$ \\
\hline 2 & 0.952 & $3.93 \times 10^{-2}$ & $2.09 \times 10^{-2}$ & 2 & 0.942 & $3.24 \times 10^{-2}$ & $2.09 \times 10^{-2}$ \\
\hline 3 & 0.948 & $4.36 \times 10^{-2}$ & $2.03 \times 10^{-2}$ & 3 & 0.946 & $3.63 \times 10^{-2}$ & $2.15 \times 10^{-2}$ \\
\hline 4 & 0.948 & $4.20 \times 10^{-2}$ & $2.05 \times 10^{-2}$ & 4 & 0.95 & $3.08 \times 10^{-2}$ & $2.39 \times 10^{-2}$ \\
\hline 5 & 0.95 & $4.29 \times 10^{-2}$ & $2.07 \times 10^{-2}$ & 5 & 0.944 & $3.55 \times 10^{-2}$ & $2.90 \times 10^{-2}$ \\
\hline
\end{tabular}

Table 34. Results of interval predictions for data generated from ST-GARCH(1,1).

\begin{tabular}{|c|c|c|c|c|c|c|c|}
\hline \multicolumn{4}{|c|}{ L2 } & \multicolumn{4}{|c|}{ L1 } \\
\hline \multicolumn{4}{|c|}{ GARCH(1,1) } & \multicolumn{4}{|c|}{$\operatorname{GARCH}(1,1)$} \\
\hline STEPS & CVR & LEN & ST.ERR & STEPS & CVR & LEN & ST.ERR \\
\hline 1 & 0.89 & $2.80 \times 10^{-3}$ & $2.14 \times 10^{-3}$ & 1 & 0.894 & $2.53 \times 10^{-3}$ & $3.80 \times 10^{-3}$ \\
\hline 2 & 0.888 & $2.19 \times 10^{-3}$ & $2.79 \times 10^{-3}$ & 2 & 0.902 & $2.88 \times 10^{-3}$ & $4.28 \times 10^{-3}$ \\
\hline 3 & 0.904 & $2.15 \times 10^{-3}$ & $2.87 \times 10^{-3}$ & 3 & 0.884 & $2.48 \times 10^{-3}$ & $3.20 \times 10^{-3}$ \\
\hline 4 & 0.908 & $2.07 \times 10^{-3}$ & $2.04 \times 10^{-3}$ & 4 & 0.9012 & $2.73 \times 10^{-3}$ & $4.21 \times 10^{-3}$ \\
\hline 5 & 0.896 & $2.00 \times 10^{-3}$ & $2.09 \times 10^{-3}$ & 5 & 0.89 & $2.02 \times 10^{-3}$ & $4.92 \times 10^{-3}$ \\
\hline \multicolumn{4}{|c|}{ EXP-NoVaS } & \multicolumn{4}{|c|}{ EXP-NoVaS } \\
\hline STEPS & CVR & LEN & ST.ERR & STEPS & CVR & LEN & ST.ERR \\
\hline 1 & 0.956 & $3.35 \times 10^{-3}$ & $2.57 \times 10^{-3}$ & 1 & 0.958 & $3.70 \times 10^{-3}$ & $2.69 \times 10^{-3}$ \\
\hline 2 & 0.96 & $3.40 \times 10^{-3}$ & $2.58 \times 10^{-3}$ & 2 & 0.944 & $3.60 \times 10^{-3}$ & $2.54 \times 10^{-3}$ \\
\hline 3 & 0.946 & $3.60 \times 10^{-3}$ & $2.73 \times 10^{-3}$ & 3 & 0.966 & $3.64 \times 10^{-3}$ & $2.65 \times 10^{-3}$ \\
\hline 4 & 0.942 & $3.42 \times 10^{-3}$ & $2.51 \times 10^{-3}$ & 4 & 0.956 & $3.52 \times 10^{-3}$ & $2.46 \times 10^{-3}$ \\
\hline 5 & 0.944 & $3.56 \times 10^{-3}$ & $2.70 \times 10^{-3}$ & 5 & 0.962 & $3.66 \times 10^{-3}$ & $2.53 \times 10^{-3}$ \\
\hline \multicolumn{4}{|c|}{ Simple-NoVaS } & \multicolumn{4}{|c|}{ Simple-NoVaS } \\
\hline STEPS & CVR & LEN & ST.ERR & STEPS & CVR & LEN & ST.ERR \\
\hline 1 & 0.952 & $3.44 \times 10^{-3}$ & $2.32 \times 10^{-3}$ & 1 & 0.944 & $3.44 \times 10^{-3}$ & $2.75 \times 10^{-3}$ \\
\hline 2 & 0.956 & $3.49 \times 10^{-3}$ & $2.24 \times 10^{-3}$ & 2 & 0.938 & $3.65 \times 10^{-3}$ & $2.75 \times 10^{-3}$ \\
\hline 3 & 0.958 & $3.46 \times 10^{-3}$ & $2.04 \times 10^{-3}$ & 3 & 0.934 & $3.67 \times 10^{-3}$ & $2.81 \times 10^{-3}$ \\
\hline 4 & 0.954 & $3.40 \times 10^{-3}$ & $2.10 \times 10^{-3}$ & 4 & 0.946 & $3.60 \times 10^{-3}$ & $2.72 \times 10^{-3}$ \\
\hline 5 & 0.946 & $3.73 \times 10^{-3}$ & $2.19 \times 10^{-3}$ & 5 & 0.938 & $3.51 \times 10^{-3}$ & $2.51 \times 10^{-3}$ \\
\hline \multicolumn{4}{|c|}{ GS-NoVaS } & \multicolumn{4}{|c|}{ GS-NoVaS } \\
\hline STEPS & CVR & LEN & ST.ERR & STEPS & CVR & LEN & ST.ERR \\
\hline 1 & 0.952 & $3.49 \times 10^{-3}$ & $2.18 \times 10^{-3}$ & 1 & 0.946 & $3.73 \times 10^{-3}$ & $2.16 \times 10^{-3}$ \\
\hline 2 & 0.956 & $3.30 \times 10^{-3}$ & $2.29 \times 10^{-3}$ & 2 & 0.956 & $3.86 \times 10^{-3}$ & $2.31 \times 10^{-3}$ \\
\hline 3 & 0.948 & $3.51 \times 10^{-3}$ & $2.29 \times 10^{-3}$ & 3 & 0.954 & $3.84 \times 10^{-3}$ & $2.27 \times 10^{-3}$ \\
\hline 4 & 0.948 & $3.54 \times 10^{-3}$ & $2.36 \times 10^{-3}$ & 4 & 0.95 & $3.82 \times 10^{-3}$ & $2.15 \times 10^{-3}$ \\
\hline 5 & 0.944 & $3.56 \times 10^{-3}$ & $2.70 \times 10^{-3}$ & 5 & 0.946 & $3.76 \times 10^{-3}$ & $2.22 \times 10^{-3}$ \\
\hline \multicolumn{4}{|c|}{ GE-NoVaS } & \multicolumn{4}{|c|}{ GE-NoVaS } \\
\hline STEPS & CVR & LEN & ST.ERR & STEPS & CVR & LEN & ST.ERR \\
\hline 1 & 0.946 & $3.37 \times 10^{-3}$ & $2.10 \times 10^{-3}$ & 1 & 0.954 & $3.64 \times 10^{-3}$ & $2.60 \times 10^{-3}$ \\
\hline 2 & 0.944 & $3.51 \times 10^{-3}$ & $2.76 \times 10^{-3}$ & 2 & 0.952 & $3.60 \times 10^{-3}$ & $2.55 \times 10^{-3}$ \\
\hline 3 & 0.946 & $3.60 \times 10^{-3}$ & $2.73 \times 10^{-3}$ & 3 & 0.956 & $3.61 \times 10^{-3}$ & $2.55 \times 10^{-3}$ \\
\hline 4 & 0.948 & $3.44 \times 10^{-3}$ & $2.49 \times 10^{-3}$ & 4 & 0.95 & $3.76 \times 10^{-3}$ & $2.68 \times 10^{-3}$ \\
\hline 5 & 0.948 & $3.69 \times 10^{-3}$ & $2.61 \times 10^{-3}$ & 5 & 0.944 & $3.46 \times 10^{-3}$ & $2.31 \times 10^{-3}$ \\
\hline
\end{tabular}


Table 35. Results of interval predictions for data generated from SV-GARCH(1,1).

\begin{tabular}{|c|c|c|c|c|c|c|c|}
\hline \multicolumn{4}{|c|}{ L2 } & \multicolumn{4}{|c|}{ L1 } \\
\hline \multicolumn{4}{|c|}{ GARCH $(1,1)$} & \multicolumn{4}{|c|}{ GARCH $(1,1)$} \\
\hline STEPS & CVR & LEN & ST.ERR & STEPS & CVR & LEN & ST.ERR \\
\hline 1 & 0.878 & $2.24 \times 10^{-2}$ & $2.99 \times 10^{-2}$ & 1 & 0.884 & $1.22 \times 10^{-2}$ & $1.93 \times 10^{-2}$ \\
\hline 2 & 0.862 & $2.11 \times 10^{-2}$ & $2.51 \times 10^{-2}$ & 2 & 0.882 & $1.28 \times 10^{-2}$ & $2.10 \times 10^{-2}$ \\
\hline 3 & 0.896 & $2.49 \times 10^{-2}$ & $2.78 \times 10^{-2}$ & 3 & 0.872 & $1.17 \times 10^{-2}$ & $1.60 \times 10^{-2}$ \\
\hline 4 & 0.87 & $2.14 \times 10^{-2}$ & $2.18 \times 10^{-2}$ & 4 & 0.878 & $1.26 \times 10^{-2}$ & $1.46 \times 10^{-2}$ \\
\hline 5 & 0.892 & $2.32 \times 10^{-2}$ & $2.33 \times 10^{-2}$ & 5 & 0.876 & $1.33 \times 10^{-2}$ & $1.63 \times 10^{-2}$ \\
\hline \multicolumn{4}{|c|}{ EXP-NoVaS } & \multicolumn{4}{|c|}{ EXP-NoVaS } \\
\hline STEPS & CVR & LEN & ST.ERR & STEPS & CVR & LEN & ST.ERR \\
\hline 1 & 0.954 & $2.71 \times 10^{-2}$ & $2.59 \times 10^{-2}$ & 1 & 0.944 & $1.84 \times 10^{-2}$ & $2.33 \times 10^{-2}$ \\
\hline 2 & 0.952 & $2.80 \times 10^{-2}$ & $2.67 \times 10^{-2}$ & 2 & 0.93 & $2.01 \times 10^{-2}$ & $2.46 \times 10^{-2}$ \\
\hline 3 & 0.956 & $2.79 \times 10^{-2}$ & $2.66 \times 10^{-2}$ & 3 & 0.954 & $1.96 \times 10^{-2}$ & $2.45 \times 10^{-2}$ \\
\hline 4 & 0.95 & $2.84 \times 10^{-2}$ & $2.65 \times 10^{-2}$ & 4 & 0.942 & $2.19 \times 10^{-2}$ & $2.62 \times 10^{-2}$ \\
\hline 5 & 0.968 & $3.07 \times 10^{-2}$ & $2.84 \times 10^{-2}$ & 5 & 0.93 & $1.88 \times 10^{-2}$ & $2.43 \times 10^{-2}$ \\
\hline \multicolumn{4}{|c|}{ Simple-NoVaS } & \multicolumn{4}{|c|}{ Simple-NoVaS } \\
\hline TEPS & CVR & LEN & ST.ERR & STEPS & CVR & LEN & ST.ERR \\
\hline 1 & 0.952 & $2.68 \times 10^{-2}$ & $2.79 \times 10^{-2}$ & 1 & 0.942 & $2.08 \times 10^{-2}$ & $2.40 \times 10^{-2}$ \\
\hline 2 & 0.95 & $2.77 \times 10^{-2}$ & $2.92 \times 10^{-2}$ & 2 & 0.932 & $2.46 \times 10^{-2}$ & $2.53 \times 10^{-2}$ \\
\hline 3 & 0.946 & $2.78 \times 10^{-2}$ & $2.74 \times 10^{-2}$ & 3 & 0.95 & $2.63 \times 10^{-2}$ & $2.84 \times 10^{-2}$ \\
\hline 4 & 0.958 & $3.05 \times 10^{-2}$ & $2.90 \times 10^{-2}$ & 4 & 0.926 & $2.25 \times 10^{-2}$ & $2.65 \times 10^{-2}$ \\
\hline 5 & 0.954 & $2.66 \times 10^{-2}$ & $2.73 \times 10^{-2}$ & 5 & 0.934 & $2.07 \times 10^{-2}$ & $2.41 \times 10^{-2}$ \\
\hline \multicolumn{4}{|c|}{ GS-NoVaS } & \multicolumn{4}{|c|}{ GS-NoVaS } \\
\hline STEPS & CVR & LEN & ST.ERR & STEPS & CVR & LEN & ST.ERR \\
\hline 1 & 0.952 & $2.68 \times 10^{-2}$ & $2.79 \times 10^{-2}$ & 1 & 0.95 & $2.17 \times 10^{-2}$ & $2.42 \times 10^{-2}$ \\
\hline 2 & 0.95 & $2.77 \times 10^{-2}$ & $2.92 \times 10^{-2}$ & 2 & 0.96 & $2.05 \times 10^{-2}$ & $2.40 \times 10^{-2}$ \\
\hline 3 & 0.952 & $2.84 \times 10^{-2}$ & $2.96 \times 10^{-2}$ & 3 & 0.95 & $2.18 \times 10^{-2}$ & $2.44 \times 10^{-2}$ \\
\hline 4 & 0.944 & $2.28 \times 10^{-2}$ & $2.34 \times 10^{-2}$ & 4 & 0.95 & $2.22 \times 10^{-2}$ & $2.48 \times 10^{-2}$ \\
\hline 5 & 0.946 & $2.34 \times 10^{-2}$ & $2.18 \times 10^{-2}$ & 5 & 0.942 & $2.28 \times 10^{-2}$ & $2.55 \times 10^{-2}$ \\
\hline \multicolumn{4}{|c|}{ GE-NoVaS } & \multicolumn{4}{|c|}{ GE-NoVaS } \\
\hline STEPS & CVR & LEN & ST.ERR & STEPS & CVR & LEN & ST.ERR \\
\hline 1 & 0.954 & $2.71 \times 10^{-2}$ & $2.59 \times 10^{-2}$ & 1 & 0.952 & $2.02 \times 10^{-2}$ & $2.35 \times 10^{-2}$ \\
\hline 2 & 0.948 & $2.52 \times 10^{-2}$ & $2.29 \times 10^{-2}$ & 2 & 0.948 & $2.18 \times 10^{-2}$ & $2.47 \times 10^{-2}$ \\
\hline 3 & 0.956 & $2.79 \times 10^{-2}$ & $2.66 \times 10^{-2}$ & 3 & 0.948 & $1.95 \times 10^{-2}$ & $2.29 \times 10^{-2}$ \\
\hline 4 & 0.95 & $2.84 \times 10^{-2}$ & $2.65 \times 10^{-2}$ & 4 & 0.942 & $2.29 \times 10^{-2}$ & $2.58 \times 10^{-2}$ \\
\hline 5 & 0.946 & $2.74 \times 10^{-2}$ & $2.46 \times 10^{-2}$ & 5 & 0.946 & $2.10 \times 10^{-2}$ & $2.34 \times 10^{-2}$ \\
\hline
\end{tabular}

Our results accentuate the drawbacks of $\operatorname{GARCH}(1,1)$ associated with one-step ahead predictions from previous work; see Politis (2015) and the references therein. In particular, NoVaS methods were invariably more robust than $\mathrm{GARCH}(1,1)$ fitting when the data have a stochastic structure that deviates from a stationary GARCH $(1,1)$ model, e.g., a time-varying $\mathrm{GARCH}$, a $\mathrm{GARCH}$ with structure breaks, etc.

\section{Conclusions}

In this paper, we derived a new way of multi-step-ahead predictions for ARCH/GARCH and NoVaS methods only based on the basic assumptions of models or transformation. This method has good properties based on our theoretical methodology and simulated results. To sum up:

- The ARCH/GARCH version of our algorithms worked well for data that are generated by a stationary GARCH $(1,1)$ model.

- The NoVaS version of our algorithms worked well for time series data that are either GARCH or have a stochastic structure that deviates from a stationary GARCH $(1,1)$ model. 
- There was no apparent error accumulation issue in the multi-step-ahead prediction.

- The methods were theoretically and computationally straightforward.

- Combined with the one-step ahead prediction results in Politis (2015), NoVaS was shown to outperform GARCH model fitting most of the time, for $h$-step-ahead prediction for all $h \geq 1$.

Author Contributions: Conceptualization, D.N.P.; methodology, J.C. and D.N.P.; software, J.C.; validation, J.C.; formal analysis, J.C.; investigation, J.C.; resources, J.C.; data curation, J.C.; writing original draft preparation, J.C.; writing review and editing, D.N.P.; visualization, J.C.; supervision, D.N.P.; project administration, D.N.P.; funding acquisition, D.N.P.

Funding: This research was partially supported by the National Science Foundation Grant DMS 16-13026.

Conflicts of Interest: The authors declare no conflict of interest. The funders had no role in the design of the study; in the collection, analyses, or interpretation of data; in the writing of the manuscript; nor in the decision to publish the results.

\section{References}

Abadir, Karim, Alessandra Luati, and Paolo Paruolo. 2018. The Forecast Density of a Garch(1,1). Working Paper. Bologna: University of Bologna.

Andersen, Torben G., Tim Bollerslev, Peter F. Christoffersen, and Francis X. Diebold. 2006. Volatility and Correlation Forecasting. In Handbook of Economic Forecasting. Amsterdam: Elsevier, vol. 1, chp. 15, pp. 777-878.

Bollerslev, Tim, Ray Y. Chou, and Kenneth F. Kroner. 1992. ARCH modeling in finance: A review of the theory and empirical evidence. Journal of Econometrics 52: 5-59. [CrossRef]

Bose, Arup, and Kanchan Mukherjee. 2009. Bootstrapping a weighted linear estimator of the arch parameters. Journal of Time Series Analysis 30: 315-31. doi:10.1111/j.1467-9892.2009.00613.x. [CrossRef]

Engle, Robert F. 1982. Autoregressive conditional heteroscedasticity with estimates of the variance of united kingdom inflation. Econometrica 50: 987-1007. [CrossRef]

Francq, Christian, and Jean-Michel Zakoian. 2011. GARCH Models: Structure, Statistical Inference and Financial Applications. Chichester: John Wiley \& Sons.

Pan, Li, and Dimitris N. Politis. 2014. Bootstrap prediction intervals for markov processes. Computational Statistics $\mathcal{E}$ Data Analysis 100: 467-94.

Pan, Li, and Dimitris N. Politis. 2016. Bootstrap prediction intervals for linear, nonlinear and nonparametric autoregressions. Journal of Statistical Planning and Inference 177: 1-27. [CrossRef]

Politis, Dimitris N. 2003. A normalizing and variance-stabilizing transformation for financial time series. In Recent Advances and Trends in Nonparametric Statistics. Edited by Michael G. Akritas and Dimitris N. Politis. Amsterdam: JAI, pp. 335-47.doi:10.1016/B978-044451378-6/50022-3. [CrossRef]

Politis, Dimitris N. 2007. Model-free versus Model-based Volatility Prediction. Journal of Financial Econometrics 5: 358-59. doi:10.1093/jjfinec/nbm004. [CrossRef]

Politis, Dimitris N. 2015. Model-Free Prediction and Regression: A Transformation-Based Approach to Inference. New York: Springer.

(C) 2019 by the authors. Licensee MDPI, Basel, Switzerland. This article is an open access article distributed under the terms and conditions of the Creative Commons Attribution (CC BY) license (http:// creativecommons.org/licenses/by/4.0/). 